\title{
The Effect of Acute Aerobic Exercise on the Consolidation of Motor Memories
}

by

Sarah Holman

\author{
A thesis \\ presented to the University of Waterloo \\ in fulfillment of the \\ thesis requirement for the degree of \\ Master of Science \\ in \\ Kinesiology
}

Waterloo, Ontario, Canada, 2019

(C) Sarah Holman 2019 


\section{Author's Declaration}

I hereby declare that I am the sole author of this thesis. This is a true copy of the thesis, including any required final revisions, as accepted by my examiners.

I understand that my thesis may be made electronically available to the public. 


\begin{abstract}
Previous research has shown that acute aerobic exercise performed prior to motor training can assist with motor skill acquisition through enhancement of motor cortical plasticity.

Recently, studies using high intensity interval training performed post-motor training have found improvements in the retention of the motor skill. This suggests that exercise performed postmotor training may enhance motor memory consolidation, although the mechanisms of this are unclear. We hypothesized that acute continuous moderate intensity exercise performed postmotor training would also assist with motor skill retention and that this behavioural change would be positively correlated with neural markers of cortical plasticity. Thirty-three participants were randomly assigned to one of two groups: exercise (EXE) or control (CON). During the first visit, participants completed a motor training session of a bimanual wrist flexion task using wrist movements to control the cursor position on a computer screen to reach a target. Movement to the target was cued, allowing for the generation of a cortical movement-related potential (MRP). MRP modulations represent changes in the excitability of the brain prior to movement which are associated with task performance. After motor training, EXE performed a session of continuous moderate intensity exercise on a recumbent bike for 20 minutes $(70 \%$ of heart rate reserve (HRR)). CON read for the same amount of time. Both groups completed two post-training tests after the exercise or rest: one 10 minutes after the exercise/rest session (post-training test 1), and one once heart rate returned to resting level in EXE (post-training test 2) or 30 minutes after rest in CON. Participants returned to the lab 1 day and 7 days later to complete retention and transfer tests of the task. MRPs were measured using electroencephalography (EEG) to investigate neural markers related to motor performance and exercise during the first visit. To assess behavioural changes measures of speed and accuracy were collected at all timepoints as response time (RT)
\end{abstract}


and root mean square (RMS) of the difference in the actual from the ideal trajectory of the cursor.

Results show that EXE had a smaller change in accuracy scores compared to CON at both retention timepoints, however this group difference was only significant at the 7 day retention. There were no significant differences in speed between the groups at the retention tests and no significant differences in accuracy or speed between the groups at the transfer tests. Consistent with previous motor training studies our MRP data shows an increase in amplitude from early to late training, however this was only significant at $\mathrm{CZ}$ and not FCZ. MRP amplitude was not significantly increased after exercise at the post-training test 2 . Correlational analysis revealed a significant correlation between the change in the MRP amplitude sum from early to late training and the change in RT from the last 5 trials of post-training 2 to retention 1 . This was only observed in EXE and when both groups were pooled together. Our results suggest that postmotor training exercise helps to retain the accuracy of the skill after motor training. Additionally, there may be a relationship between excitability increases during training and performance of the skill at retention which may be enhanced with post-motor training exercise. These results inform motor learning paradigms and future studies with other populations including older adults and neurorehabilitation patients. 


\section{Acknowledgements}

I would not have been able to complete this thesis without the following people.

First, Dr. Richard Staines, thank you for your constant support and mentorship. You are an incredible supervisor and I am so grateful to have studied under your guidance for the past 2 years. To my committee members, Dr. Laura Middleton and Dr. Sean Meehan, thank you for providing me with different perspectives. Your questions and suggestions helped to make my project and this document something I am proud of.

To my lab mates and peers, thank you for sharing your advice and expertise. I am lucky to have been surrounded by such intelligent, supportive, and encouraging individuals.

To my family, thank you for always believing in me. I appreciate everything each of you has done to support me throughout my education. I could not ask for better parents or siblings. To Zen, you live up to your name, thank you for keeping me grounded. 


\section{Table of Contents}

List of Figures................................................................................................................................................... viii

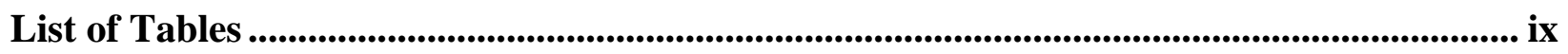

List of Abbreviations ............................................................................................................................. $\mathrm{x}$

1.0 Introduction.................................................................................................................................................... 1

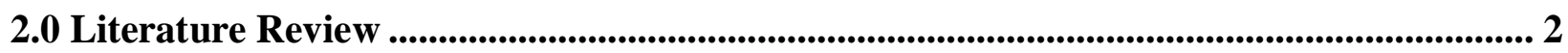

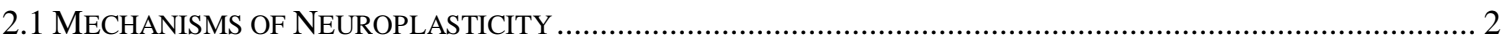

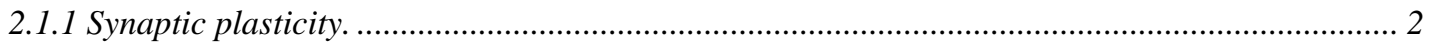

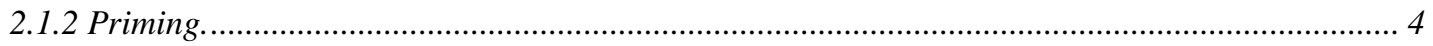

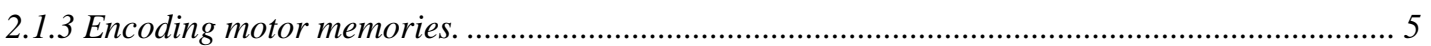

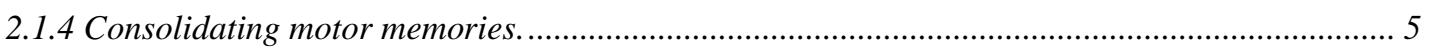

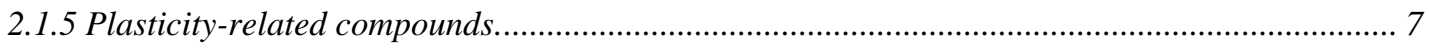

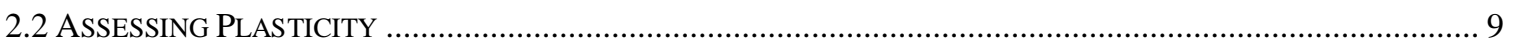

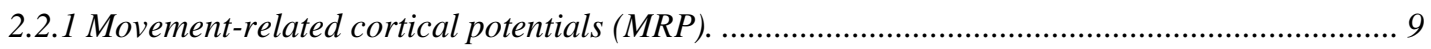

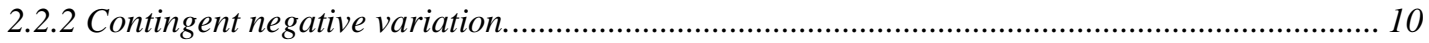

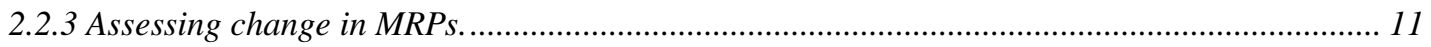

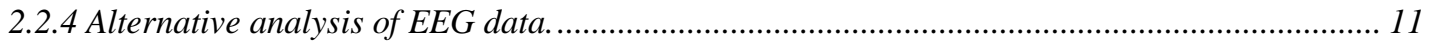

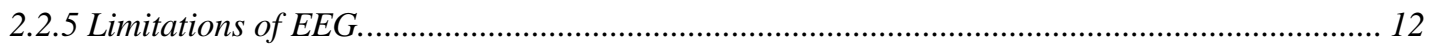

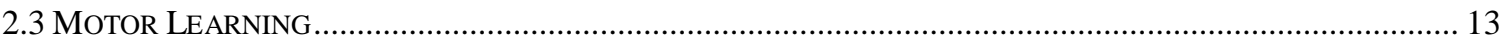

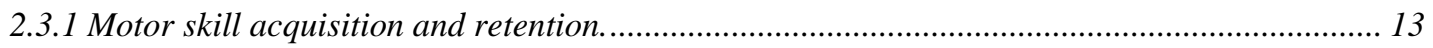

2.3.2 Behavioural changes associated with motor learning............................................................... 15

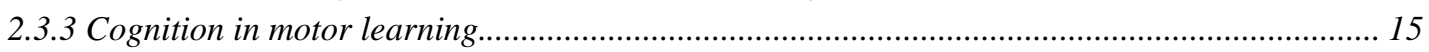

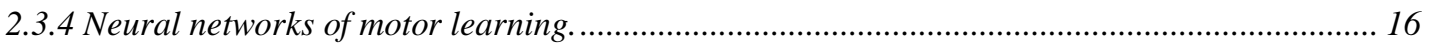

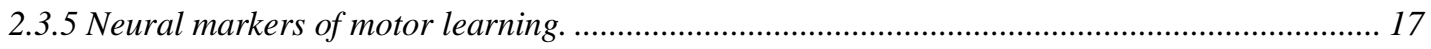

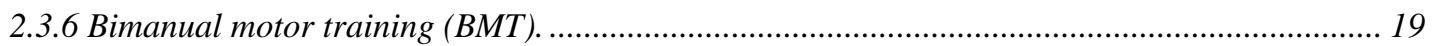

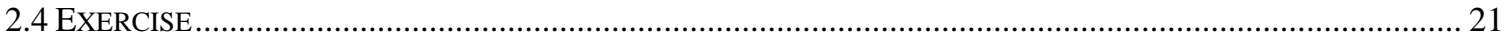

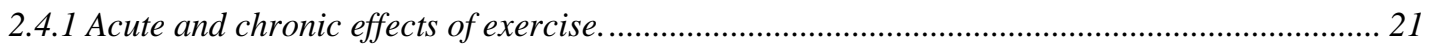

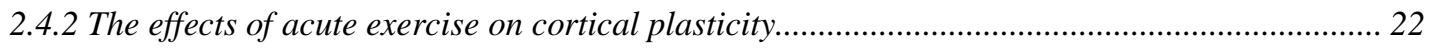

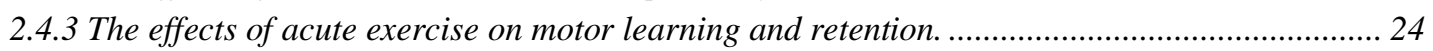

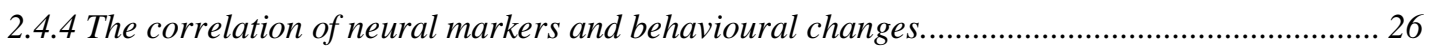

3.0 Rationale, Objectives, and Hypotheses .......................................................................... 29

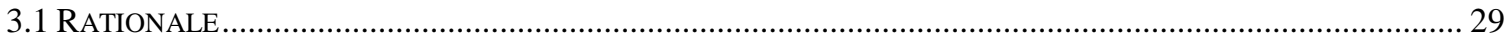

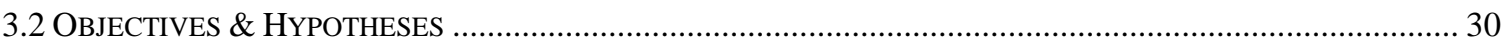

4.0 Methods.................................................................................................................................................. 32

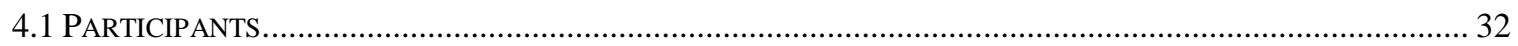

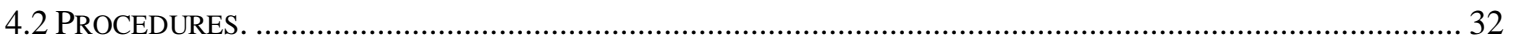

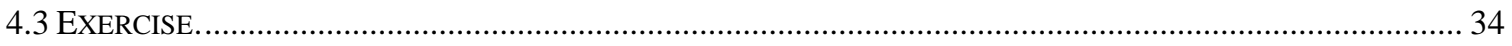

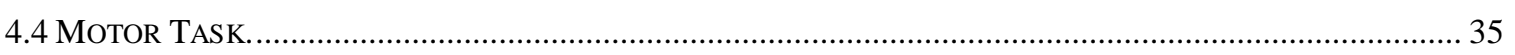

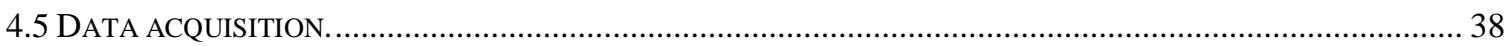




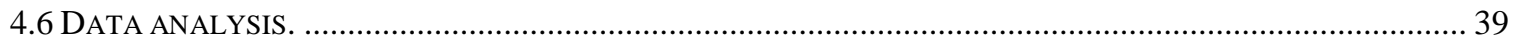

5.0 Proposed Hypotheses Testing .......................................................................................................... 42

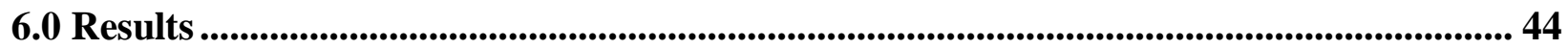

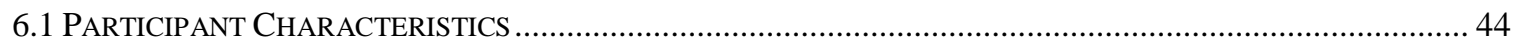

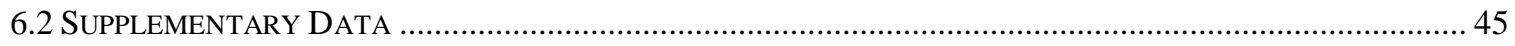

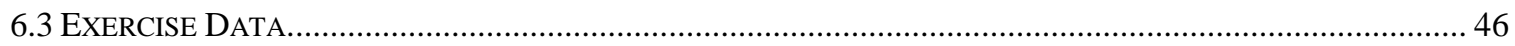

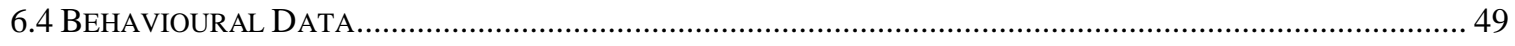

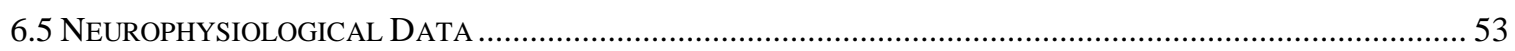

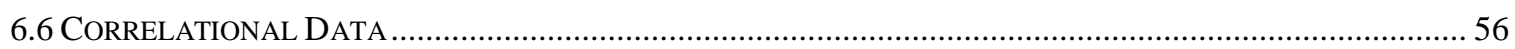

7.0 Discussion ........................................................................................................................................................... 58

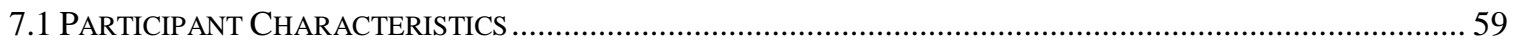

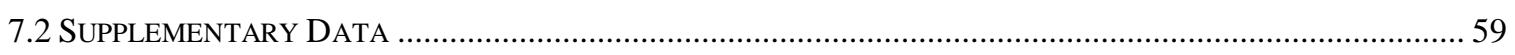

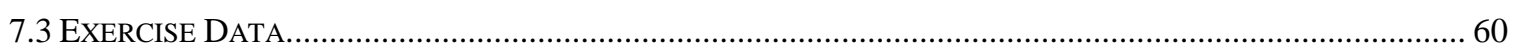

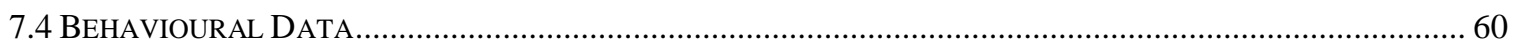

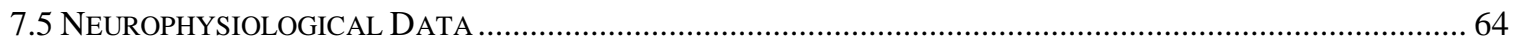

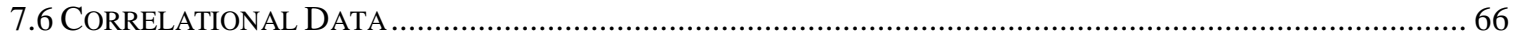

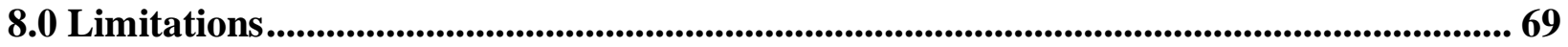

9.0 Conclusions and Future Directions ............................................................................................... 70

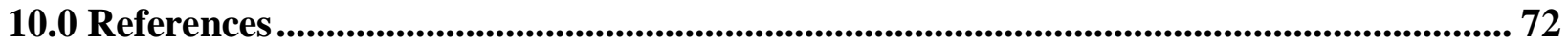




\section{List of Figures}

Figure 1

Figure 2

Figure 3

Figure 4

Figure 5

50

Figure 6

Figure 7

Figure 8

53

Figure 9

54

Figure 10

54

Figure 11

55

Figure 12

55

Figure 13 


\section{List of Tables}

Table 1

45

Table 2 


\section{List of Abbreviations}

\begin{tabular}{|c|c|}
\hline AMPA & Alpha-amino-3-hydroxy-5-methyl-4-isoxazolepropionic acid \\
\hline BDNF & Brain derived neurotropic factor \\
\hline BMI & Body mass index \\
\hline BMT & Bimanual motor training \\
\hline BOLD & Blood-oxygen-level dependent \\
\hline $\mathrm{BP}$ & Bereitschaftspotential \\
\hline $\mathrm{Ca}^{2+}$ & Calcium \\
\hline CMC & Corticomuscular coherence \\
\hline $\mathrm{CNV}$ & Contingent negative variation \\
\hline $\mathrm{CON}$ & Control group \\
\hline cTBS & Continuous theta burst stimulation \\
\hline DA & Dopamine \\
\hline DLPFC & Dorsolateral-prefrontal cortex \\
\hline ECR & Extensor carpi radialis \\
\hline EEG & Electroencephalography \\
\hline EHQ & Edinburgh Handedness Questionnaire \\
\hline E-LTP & Early long term potentiation \\
\hline EMG & Electromyography \\
\hline EPI & Epinephrine \\
\hline EPSP & Excitatory postsynaptic potential \\
\hline ERD & Event-related desynchronization \\
\hline EXE & Exercise group \\
\hline FCR & Flexor carpi radialis \\
\hline fMRI & Functional magnetic resonance imaging \\
\hline GABA & Gamma-aminobutyric acid \\
\hline $\mathrm{GABA}_{\mathrm{A}} / \mathrm{GABA}_{\mathrm{B}}$ & Subtype of gamma-aminobutyric acid \\
\hline GAQ & Get Active Questionnaire \\
\hline HR & Heart rate \\
\hline HRR & Heart rate reserve \\
\hline ICF & Intracortical facilitation \\
\hline IGF-1 & Insulin-like growth factor 1 \\
\hline IPAQ & International Physical Activity Questionnaire \\
\hline iTBS & Intermittent theta burst stimulation \\
\hline LICI & Long-interval intracortical inhibition \\
\hline L-LTP & Late long term potentiation \\
\hline LTD & Long term depression \\
\hline LTP & Long term potentiation \\
\hline M1 & Primary motor cortex \\
\hline MEP & Motor evoked potential \\
\hline MET & Metabolic equivalent \\
\hline mRNA & Messenger ribonucleic acid \\
\hline MRP & Movement-related cortical potential \\
\hline $\mathrm{Mg}^{2+}$ & Magnesium \\
\hline $\mathrm{Na}^{+}$ & Sodium \\
\hline
\end{tabular}




$\begin{array}{ll}\text { NE } & \text { Norepinephrine } \\ \text { NMDA } & N \text {-methyl-D-aspartate } \\ \mathrm{O}_{2} & \text { Oxygen } \\ \text { PANAS } & \text { Positive and Negative Affect Schedule } \\ \text { PAS } & \text { Paired associative stimulation } \\ \text { PFC } & \text { Prefrontal cortex } \\ \text { PMC } & \text { Premotor cortex } \\ \text { RAP } & \text { Re-afferent potential } \\ \text { RMS } & \text { Root mean square } \\ \text { RPE } & \text { Rate of perceived exertion } \\ \text { RPM } & \text { Revolutions per minute } \\ \text { RT } & \text { Response time } \\ \text { rTMS } & \text { Repetitive transcranial magnetic stimulation } \\ \text { S1 } & \text { Primary somatosensory cortex } \\ \text { SICI } & \text { Short-interval intracortical inhibition } \\ \text { SMA } & \text { Supplementary motor area } \\ \text { SMHSQ } & \text { St. Mary's Hospital Sleep Questionnaire } \\ \text { SSS } & \text { Stanford Sleepiness Scale } \\ \text { tDCS } & \text { Transcranial direct current stimulation (tDCS) } \\ \text { TMS } & \text { Transcranial magnetic stimulation } \\ \text { VEGF } & \text { Vascular endothelial growth factor }\end{array}$




\subsection{Introduction}

The ability of humans to learn new motor skills is vital to our survival. Motor skills allow us to interact with the external environment, live independently, and engage in recreational activities. Across the average human's lifespan there are scenarios in which new motor skills need to be learned. Research has helped to inform motor learning paradigms including strategies to enhance motor learning before, during, and after motor training. The time period after practice is important for the consolidation of the motor memory, which is the encoded sequence of muscle activations, and depending on what occurs during this time, retention of the motor skill can be enhanced or diminished. One such strategy shown to enhance the consolidation of motor memories is an acute session of high intensity interval training (Roig, Skriver, Lundbye-Jensen, Kiens, \& Nielsen, 2012). The purpose of this study was to examine whether continuous moderate intensity post-motor training exercise would assist with the retention of a bimanual motor skill. A secondary objective of the study was to investigate the neurophysiological changes in the brain during and post-motor training in an attempt to correlate the hypothesized behavioural improvement with neural markers of motor learning and consolidation. 


\subsection{Literature Review}

\subsection{Mechanisms of Neuroplasticity}

2.1.1 Synaptic plasticity. Neuroplasticity is the ability of the brain to adapt in response to stimuli. There are various forms of neuroplasticity, one form commonly studied is synaptic plasticity, in which the adaptation occurs at the level of the synapse. This process is thought to be an underlying mechanism of many types of learning and memory, including that of motor skills. Two types of synaptic plasticity are long term potentiation (LTP) and long term depression (LTD). LTP was first discovered in the rabbit hippocampus and is the strengthening of the synaptic connections through high-frequency signals (Lømo, 1966). LTD is the weakening of synaptic connections through low frequency signals or through the temporal unpairing of a stimulus with an action potential (Ito \& Kano, 1982). These processes are input-specific, meaning that they only affect the synapses that are activated and not all synapses on the involved neurons. Other properties of LTP and LTD include associativity, and cooperativity. Associativity describes the phenomenon that although a weak stimulation in isolation will not induce LTP, when a weak stimulation is paired with a strong stimulation from a different presynaptic neuron, both of the synapses will be involved in LTP. Cooperativity involves a similar process; however, it occurs when multiple weak stimuli converge onto a single postsynaptic cell. The sum of these weak stimuli produce a stimulus strong enough to induce LTP in the postsynaptic cell.

LTP and LTD occur as a result of ionotropic glutamate receptor activity: $N$-methyl-Daspartate (NMDA), and alpha-amino-3-hydroxy-5-methyl-4-isoxazolepropionic acid (AMPA). In LTP, both the pre- and postsynaptic neurons must be active (depolarized). This depolarization is the result of glutamate being released in the synaptic cleft. Glutamate binds to both NMDA and AMPA receptors on the postsynaptic cell. When this occurs, AMPA opens and allows sodium 
$\left(\mathrm{Na}^{+}\right)$into the cell, causing excitatory postsynaptic potentials (EPSPs) reflective of depolarization in the postsynaptic cell. This depolarization removes the magnesium $\left(\mathrm{Mg}^{2+}\right)$ block on the NMDA receptor pore on the postsynaptic cell, allowing the flow of ions in. Calcium $\left(\mathrm{Ca}^{2+}\right)$ and $\mathrm{Na}^{+}$enter the postsynaptic cell through the NMDA receptor and activate a secondmessenger pathway that causes the addition of more AMPA receptors into the postsynaptic membrane, allowing more glutamate to bind to these newly available receptors. This results in a larger postsynaptic response the next time glutamate is released into the synapse. This process is called early LTP (E-LTP). With enough stimulation, late LTP (L-LTP) results in the addition of a new dendritic spine which requires genetic modifications and protein synthesis signalled through second-messenger systems. The transition from E-LTP to L-LTP is thought to occur as a result of a tagging process, termed the synaptic tagging hypothesis (Frey \& Morris, 1998). Specifically, initial changes within the cell "tag" a synapse to eventually undergo L-LTP (Redondo \& Morris, 2011). Researchers have suggested that increases in levels of neurotransmitters post-exercise may assist this tagging process (Thomas, Beck, et al., 2016). LTD is the opposing process of LTP, resulting in the removal of AMPA receptors from the postsynaptic cell and the reduction of dendritic spines.

Depending on the duration and intensity of the stimuli and the environment that the subject occupies, LTP and LTD can last from hours to months. In humans, LTP and LTD are difficult to explore directly however, there is evidence that interventions such as transcranial magnetic stimulation (TMS) cause LTP and LTD-like plasticity in the primary motor cortex (M1) (Huang, Chen, Rothwell, \& Wen, 2007; Ziemann, Iliac', Pauli, Meintzschel, \& Ruge, 2004). LTP and LTD-like plasticity are also thought to occur in response to motor training (Bütefisch et al., 2000; Nudo, 2003) and can be enhanced through different exercise intensities 
(Mang, Snow, Campbell, Ross, \& Boyd, 2014; McDonnell, Buckley, Opie, Ridding, \& Semmler, 2013; Singh, Neva, \& Staines, 2014).

2.1.2 Priming. Priming refers to any action that increases the potential for neuroplasticity to occur. Exercise is thought to prime the cortex for synaptic plasticity, the specific mechanisms of which are not fully understood. It has been proposed that exercise acts to lower the threshold for the induction of LTP by increasing the availability of LTP-related compounds (Farmer et al., 2004). For example, acute exercise increases levels of peripheral brain derived neurotropic factor (BDNF) (Skriver et al., 2014; Winter et al., 2007). BDNF is a neurotrophin that supports growth of new synapses. As described below, BDNF and other compounds are involved in the various stages of LTP and may assist LTP through the described mechanisms. Increases in BDNF may also increase the likelihood for LTP to occur by decreasing inhibition in M1. This reduction of inhibition is observed in TMS studies that have shown that a single session of moderate intensity exercise results in decreases in short-interval intracortical inhibition (SICI) (Singh, Duncan, Neva, \& Staines, 2014; Singh, Neva, et al., 2014; Smith, Goldsworthy, Garside, Wood, \& Ridding, 2014). Cortical inhibition is mediated by gamma-aminobutyric acid (GABA), an inhibitory neurotransmitter. A subtype of GABA receptors, GABAA receptors, are thought to mediate SICI (Sanger, Garg, \& Chen, 2001). Animal research has discovered that in the hippocampus, $\mathrm{BDNF}$ reduces the amount of postsynaptic $\mathrm{GABA}_{\mathrm{A}}$ receptors, which causes a reduction in the amplitude of postsynaptic inhibitory currents (Brünig, Penschuck, Berninger, Benson, \& Fritschy, 2001). Therefore, one mechanism through which exercise may prime M1 for LTP-like plasticity is by increasing BDNF levels which reduces the presentation of postsynaptic $\mathrm{GABA}_{\mathrm{A}}$ receptors, thereby reducing intracortical inhibition. While most of the BDNF levels measured in humans post-exercise are peripheral, there is evidence to suggest that 
peripheral BDNF is positively correlated with brain BDNF levels (Klein et al., 2011). As well, after exercise the increase in peripheral BDNF is likely driven by an increase in brain BDNF (Rasmussen et al., 2009).

2.1.3 Encoding motor memories. Exercise performed prior to motor training has been shown to benefit motor acquisition (Statton, Encarnacion, Celnik, \& Bastian, 2015). This is thought to be achieved through priming encoding mechanisms. Encoding is the initial formation of the motor memory, which mechanistically aligns with a short-term change at the synapse, ELTP. Research examining the effects of cortical stimulation on motor memory has shown that TMS enhances the encoding of motor memories through LTP-like mechanisms (Butefisch, Khurana, Kopylev, \& Cohen, 2004). Animal studies have found associations between a decrease in the threshold for synaptic plasticity to occur and improvements in memory encoding (Stackman et al., 2002). This suggests that exercise performed before motor training may prime the central nervous system for the encoding of the motor memory. It is also possible that exercise performed before motor acquisition can assist with the consolidation of motor memories. Based on past studies, only high intensity and not moderate intensity exercise performed pre-motor training has a beneficial effect on both acquisition and retention scores (Mang et al., 2014; Roig et al., 2012; Snow et al., 2016; Statton et al., 2015). This suggests that high intensity exercise may increase LTP-related compounds (described below) long enough to affect consolidation mechanisms (Roig et al., 2016; Statton et al., 2015).

2.1.4 Consolidating motor memories. On the other hand, when exercise is performed after motor practice, it assists with the consolidation of motor memories (Roig et al., 2016, 2012; Thomas et al., 2016; Thomas, Beck, et al., 2016; Thomas, Johnsen, et al., 2016). Consolidation is when the encoded sequence is solidified and stabilized. It has been proposed that declarative 
memories, memories of facts and events, are consolidated at two levels: synaptic consolidation and systems consolidation (Dudai, 2012). Synaptic consolidation is a quick process, taking minutes to hours, and lasting hours. L-LTP is thought to be the mechanism behind consolidation at the synaptic level. Systems consolidation is a longer process, taking between days to years, involving a shift in the storage of a memory trace in the brain. Although these concepts were first proposed for declarative memory, it is possible that these forms of consolidation are also present in procedural memory of skills and tasks, including motor memories. It is likely that the procedural processes have the same mechanisms, just in different areas of the brain (BrashersKrug, Shadmehr, \& Bizzi, 1996) such as M1 (Muellbacher et al., 2002). In animals, some research has suggested that the formation of new dendritic spines (L-LTP) can occur in the contralateral M1 after just one motor training session (Xu et al., 2009), however other researchers have only found synaptogenesis in M1 after multiple days of motor training sessions (Kleim et al., 2004). In humans, increases in corticospinal excitability in M1 are positively associated with improvements in retention, leading researchers to suggest that corticospinal excitability acts as a physiological sign of consolidation (Tunovic, Press, \& Robertson, 2014). Correlations between corticospinal excitability and LTP suggest that LTP may be a mechanism of consolidation (Cantarero, Tang, O’Malley, Salas, \& Celnik, 2013; Ziemann et al., 2004). Exercise has recently been shown to enhance corticospinal excitability indicating that it may assist motor memory consolidation through these mechanisms (Ostadan et al., 2016). M1 is not the only cortical area that is involved in the consolidation of motor memories. It is likely that consolidation involves the whole sensorimotor network, including motor planning regions such as the dorsal premotor cortex (Meehan et al., 2013), and sometimes cognitive areas including the dorsolateral-prefrontal cortex (DLPFC) (Kantak, Sullivan, Fisher, Knowlton, \& Winstein, 2010, 
2011). Evidence for systems consolidation occurring for motor memories includes brain imaging studies suggesting that after a motor skill is acquired, the cortex is reorganized and the motor memory trace is stored in different areas of the brain (Shadmehr \& Holcomb, 1997).

2.1.5 Plasticity-related compounds. A few biomarkers related to exercise that have previously been studied and associated with LTP include BDNF, vascular endothelial growth factor (VEGF), insulin-like growth factor 1 (IGF-1), lactate, epinephrine (EPI), norepinephrine (NE), and dopamine (DA). Although the exact mechanisms of BDNF are not completely understood, it is believed that BDNF promotes the induction of LTP. Researchers have suggested that BDNF facilitates E-LTP (Lu, Christian, \& Lu, 2008) based on research showing that BDNF assists with the movement of synaptic vesicles to the synaptic cleft (Pozzo-Miller et al., 1999). In L-LTP, BDNF affects signalling mechanisms leading to gene transcription (Ying et al., 2002). Other studies have provided evidence that BDNF can also support LTP through other mechanisms which researchers suggest may be specific to the brain region (Panja \& Bramham, 2014). VEGF is thought to promote LTP through increasing the intracellular release of $\mathrm{Ca}^{2+}$ and activating pathways required for L-LTP (Kim et al., 2008). IGF-1 is a protein that indirectly assists with synaptic plasticity by complementing and modulating the effects of BDNF in the process of LTP (Cotman, Berchtold, \& Christie, 2007). Lactate is another compound involved as it acts as an energy substrate for LTP (Izumi, Katsuki, \& Zorumski, 1997) and increases levels of BDNF (Schiffer et al., 2011). EPI, NE, and DA are catecholamine neurotransmitters. EPI promotes the induction and increases the duration of LTP, suggesting that it assists in the conversion of E-LTP to L-LTP (Korol \& Gold, 2008). NE promotes LTP through a process termed metaplasticity. Metaplasticity is a change at the synapse that occurs before the induction of LTP/LTD making the threshold for LTP/LTD higher or lower (Abraham \& Bear, 1996). NE 
achieves this by increasing translation of messenger ribonucleic acid (mRNA) for AMPA receptor subunits (Maity, Rah, Sonenberg, Gkogkas, \& Nguyen, 2015). The effect of DA on LTP is largely related to DA receptors. When DA binds to receptors on the synaptic cells, it activates second-messenger system pathways that promote protein synthesis and genetic modifications necessary for L-LTP (Frey \& Morris, 1998). DA receptors can also modulate activity of NMDA receptors through secondary messengers and pathways in the cell (Kotecha et al., 2002). Overall, the presence of catecholamines facilitates cortical plasticity, however this relationship is not linear, but an inverted u-shape (Korol \& Gold, 2008; Monte-Silva, Liebetanz, Grundey, Paulus, \& Nitsche, 2010; Thirugnanasambandam, Grundey, Paulus, \& Nitsche, 2011).

Winter et al. (2007) measured some of these biomarkers when investigating the effect of acute high and moderate intensity aerobic exercise on vocabulary learning. Behaviourally, high intensity exercise (blood lactate level $>10 \mathrm{mmol}$ ), led to a faster learning score compared to the moderate intensity exercise $(\leq 2 \mathrm{mmol})$ or rest. The high intensity exercise group had the largest increases in BDNF, lactate, and some of the catecholamines (EPI, NE). However, moderate intensity exercise still resulted in some increases in catecholamines. There was no correlation between vocabulary learning and NE or lactate. However, in the high intensity exercise condition increases in EPI were correlated with retention scores and increases in BDNF were correlated with better acquisition. This provides some insight into how acute aerobic exercise affects BDNF and the catecholamines, and how this relationship may assist cognitive tasks such as learning and memory. Motor learning relies on different networks and areas of the brain. In a study examining the effects of aerobic exercise on motor memory, Skriver et al. (2014) found that the group that performed high intensity exercise prior to a visuomotor tracking task performed significantly better than the control group at the 24 hour and 7 day retention. Exercise increased all of the 
biomarkers, except for VEGF. A correlational analysis between performance of the skill and concentration of biomarkers revealed that NE and lactate were positively associated with acquisition rates and BDNF was positively associated with retention 1 hour and 7 days after practice. NE was also positively associated with 7 day retention scores, and lactate was positively associated with retention scores at 1 hour, 24 hours, and 7 days. This suggests that exercise improves motor memory consolidation through the elevation of LTP-related compounds.

In summary, exercise increases LTP-related compounds and reduces intracortical inhibition before LTP induction. The difference between exercise performed prior to motor training and exercise performed after motor training seems to be in the phase of plasticity it will prime. Pre-motor training exercise will increase the availability of LTP-related compounds to enhance the encoding of motor memories. Post-motor training exercise will increase the availability of LTP-related compounds to consolidate motor memories (Roig et al., 2016).

\subsection{Assessing Plasticity}

2.2.1 Movement-related cortical potentials (MRP). One way to assess plasticity is by examining changes in the electrical activity of the brain through the use of electroencephalography (EEG). One of the benefits to using EEG is that it has a high temporal resolution. EEG uses electrodes placed on the scalp to measure cortical voltage changes. In this case, synaptic plasticity is not being measured directly, but rather indirectly through accompanying changes in voltage caused by postsynaptic potentials. Such changes can be observed in MRPs (Kornhuber \& Deecke, 1965). MRPs are slow negative shifts of electrical activity that are associated with the generation of movement. MRPs occur $2 \mathrm{~s}$ before movement onset and have amplitudes of 5-30 $\mu \mathrm{V}$. Since MRPs have low frequencies of 0-5 Hz, the analysis 
of EEG data must therefore filter out higher frequencies and average many MRP traces together to account for biological noise (Luck, 2005; Wright, Holmes, \& Smith, 2011).

In the past, MRPs have been used to study motor learning (Smith \& Staines, 2006). Using EEG to measure changes to MRPs serves as an alternative marker of learning to complement behavioural performance measures. The EEG data is time-locked the onset of movement, providing a temporal window through which MRPs can be examined. MRPs can be divided into smaller components: the early component, late component, and the re-afferent potential (RAP). Each component represents a different aspect of movement planning and execution. The early component is a slow negativity that largely represents activity from the premotor and supplementary motor areas, thus it is referred to as the motor preparation stage. There are some differences in the areas contributing to this activity depending on whether the movement is selfpaced or cued. In the self-paced MRP, this early component is also known as the Bereitschaftspotential (BP), and can be further divided into two early phases. In the BP, the dominant contributing area is the supplementary motor area (SMA) and in the early component of the cued-MRP the dominant contributing area is the premotor cortex (PMC) (Smith \& Staines, 2012). The late component, representing the motor execution stage, is a sharp negativity that occurs as a result of output from the M1 via the corticospinal tract. The RAP is a positive deflection that symbolizes activity in the primary somatosensory cortex (S1) which represents feedback from the muscle spindles.

2.2.2 Contingent negative variation. The MRP is not the only negative slow wave potential to occur prior to movements. First discovered by Walter, Cooper, Aldridge, McCallum and Winter (1964), using a reaction time task, the contingent negative variation (CNV) is also associated with movement. The slow negativity of the CNV represents movement planning, 
similar to the MRP, but unlike the MRP, is representative of attending to an imperative stimulus (Brunia \& van Boxtel, 2001). Temporally, the CNV and MRP overlap, however their cortical contributions and thus their functions differ (Brunia \& van Boxtel, 2001). While the MRP is time-locked to movement onset, the CNV is time-locked to the imperative stimulus.

2.2.3 Assessing change in MRPs. Changes in MRPs are quantified through the measurement of amplitude and latency. Amplitude provides a measure of the amount of cortical activity and thus is thought to represent the effort required to plan the movement (Lang, Beisteiner, Lindinger, \& Deecke, 1992; Wright et al., 2011). The latency of the MRP indicates the length of time it takes for the MRP to start and finish and is related to the amount of time needed to plan and prepare the movement (Wright et al., 2011). There are various factors that can affect the above components of MRPs such as hand dominance, an individual's skill level, learning a motor task, and exercise, as described in later sections (Shibasaki \& Hallett, 2006; Smith \& Staines, 2010; Tarkka \& Hallett, 1990; Thacker, Middleton, McIlroy, \& Staines, 2014; Wright et al., 2011).

2.2.4 Alternative analysis of EEG data. An alternative way to assess plasticity using EEG is through spectral analysis. An EEG waveform is made up of brain waves with different frequencies. Different brain waves are dominant during certain mental states or tasks that the individual is engaged in. Using spectral analysis, the composition of the EEG waveform can be studied. Two types of brain waves are typically studied with movement-related tasks: alpha waves and beta waves. Alpha waves have a band frequency between 7.5-12.5 Hz and are dominant in an awake resting state of the brain. Beta waves have a band frequency between 12.5$30 \mathrm{~Hz}$ and are dominant during cognitive tasks. During movement, event-related desynchronization (ERD) occurs, which is a decrease in amplitude of beta and alpha waves. This 
decrease is indicative of increased brain activity and excitability in sensorimotor areas (Neuper \& Pfurtscheller, 2001). Previous research summarized by Dal Maso, Desormeau, Boudrias, and Roig (2018) demonstrate that alpha- and beta-band ERD are modulated during and post-motor training tasks. Further, research has suggested that beta-band ERD may be a neural marker for the consolidation of motor memories (Pollok, Latz, Krause, Butz, \& Schnitzler, 2014).

Coherence analysis assesses the functional connectivity of brain areas. This is beneficial when studying a network of brain regions such as the neural networks of motor learning discussed in the next section. Additionally, coherence analysis can be used to determine synchrony between activity in the two hemispheres. Using this approach, Andres et al. (1999) found that during bimanual motor training (BMT) the functional connectivity of sensorimotor areas of the right and left hemispheres increases. EEG can also be used in combination with electromyography (EMG) to assess corticomuscular coherence (CMC). CMC is a measure of the relationship between brain and muscle activity. After motor training, the amplitude of CMC increases (Perez, Lundbye-Jensen, \& Nielsen, 2006). In summary, these techniques can be used to study motor learning. Some of the above have recently been employed to measure the effects of post-motor training exercise on the consolidation of motor memories (Dal Maso et al., 2018). The results of this study are discussed below. The current study adds to this literature by analyzing modulations of MRP components.

2.2.5 Limitations of EEG. EEG is limited in that only the brain activity at the cortical level is detected. Therefore, the contributions of subcortical structures to the detected EEG signal are not able to be directly determined. Additionally, the spatial resolution of EEG is limited. Consequently, researchers cannot differentiate between areas of the brain that are active and contributing to the overall measured brain activity with specificity. 


\subsection{Motor Learning}

2.3.1 Motor skill acquisition and retention. Motor learning refers to a permanent change in a movement response that is the result of practice or experience (Schmidt \& Lee, 2011). This process results in the formation of a motor memory, which is the encoded sequence of muscle activations, causing plastic changes in the brain referred to as the motor engram (Eichenbaum, 2016). Motor learning is assessed by measuring the acquisition, retention, and transfer of motor skills. Acquisition refers to the process whereby the learner is introduced to the task and integrates cognitive and motor aspects of the task to improve their performance (Newell, 1991). Retention of a motor skill refers to the ability to perform the motor skill at a later time point. Long-term retention tests are thus more indicative of motor learning, as permanent changes must be assessed not hours post-training, but rather, days, weeks, or months later (Schmidt \& Bjork, 1992). Transfer of the motor skill is the ability to perform the skill in a new task or context (Müssgens \& Ullén, 2015). Both retention and transfer are important in neurorehabilitation protocols since the goal of such is for the individual to be able to perform the skill months and years into the future, and to be able to perform it in diverse every-day settings (Krakauer, 2006).

Different types of practice, such as constant vs. variable practice and blocked vs. random practice employed during acquisition can affect retention and transfer of the skill. Constant practice is when the learner repeatedly performs the skill without any task modifications. For example, reaching to the same target, at the same speed, from the same starting position. Variable practice is practice of the same skill with slight modifications such as reaching to different targets or using different speeds or starting positions. Variable practice can be scheduled in blocks or randomly. For example, blocked practice consists of moving to target A 
for 20 trials and then target B for 20 trials. Random practice consists of moving to randomized targets each trial, for example target A for trial 1, target B for trial 2, etc. Motor learning research has shown that variable practice leads to better retention and transfer of the motor skill than constant practice (Müssgens \& Ullén, 2015; Schmidt \& Bjork, 1992). But variable practice must be scheduled randomly to have this beneficial effect (Schmidt, 1975; Sherwood, 1996; Wulf, 1991). Random variable practice is thought to benefit motor learning through contextual interference. When different tasks or variations of a skill are completed in succession the tasks interfere with one another. While the short-term performance may be negatively affected, this greatly improves retention and transfer of the task as it requires the individual to reconstruct each motor plan every time thus resulting in a more robust motor memory than if completing the same task over and over (Müssgens \& Ullén, 2015; Wulf, 1991). This reconstruction requires cognitive engagement such as increased attention and use of problem-solving skills. It is not surprising then that constant and (random) variable practice seem to rely on different areas of the sensorimotor network to consolidate the motor skill. Variable practice dominantly relies on the DLPFC, and constant practice relies on M1 (Kantak et al., 2010). Retention and transfer performance are poor when 1-Hz repetitive TMS (rTMS) is applied to the DLPFC after variable practice. This behavioural decrement is not observed when 1-Hz rTMS is applied to M1 after variable practice. The reverse is true for constant practice - 1-Hz rTMS applied to DLPFC has no effect on retention and transfer scores, and 1-Hz rTMS applied to M1 negatively affects retention and transfer scores (Kantak et al., 2010, 2011). This difference in areas of the brain that are involved in the consolidation of the motor skill for random variable and constant practice may contribute to why random variable practice leads to better retention and transfer of the skill. 
2.3.2 Behavioural changes associated with motor learning. Fast and slow learning and online and offline learning are terms used to describe behavioural learning processes. Fast learning refers to the quick, initial improvements in the task that happen over the first training session(s). This time period of fast learning is variable depending on the difficulty of the motor task - it could be within the first training session for easier tasks or over many training sessions for more difficult tasks (Dayan \& Cohen, 2011). Slow learning refers to the performance improvements of smaller increments that are seen over a longer period of time (Doyon \& Benali, 2005). Online and offline learning refer to the changes in skill performance that occur during and after the training sessions respectively. Offline learning is the result of the neural processes of consolidation, and it allows the learner to transition from the fast to the slow learning phase (Dayan \& Cohen, 2011; Doyon \& Benali, 2005; Karni et al., 1998). Offline learning can be enhanced or diminished based on what occurs during the period immediately following motor practice. For example, sleep enhances offline motor learning (King, Hoedlmoser, Hirschauer, Dolfen, \& Albouy, 2017) and introducing an entirely new motor skill after motor training diminishes offline learning (Brashers-Krug et al., 1996; Cantarero et al., 2013).

2.3.3 Cognition in motor learning. Motor learning requires cognitive input such as attention, task-switching, inhibition of response, information processing of visuospatial cues, and working memory. These are specifically important during the early phases of motor learning when the task is initially introduced, and learners need to devote more cognitive resources to be able to complete the novel task. Increased activity in frontal cortical areas such as the prefrontal cortex (PFC) and pre-SMA represent this higher cognitive load during early motor learning (Jueptner et al., 1997; Nachev, Kennard, \& Husain, 2008; Pascual-Leone, Wassermann, Grafman, \& Hallett, 1996; Robertson, Tormos, Maeda, \& Pascual-Leone, 2001). 
2.3.4 Neural networks of motor learning. There are two sensorimotor networks of brain structures thought to be involved in fast and slow phases of motor learning, the storage of motor memories, and the sites of the motor engram. The two networks are the cortico-basal gangliathalamo-cortical (cortico-striatal) and the cortico-cerebello-thalamo-cortical (cortico-cerebellar) systems. A model initially proposed by Doyon and Ungerleider (2002) suggested that both systems are involved in the fast learning phase of motor sequence learning, when participants are required to learn a sequence of movements, and motor adaptation, when participants adapt their movement to external perturbations. In the slow learning phase of motor sequence learning and motor adaptation, different networks are responsible for the consolidation and reactivation of the motor memory. The cortico-striatal network is involved in the slow learning phase of motor sequence learning, and the cortico-cerebellar network is involved in the slow learning phase of a motor adaptation skill. Consequently, motor memories of motor sequence skills are represented in the cortico-striatal network, and motor memories of motor adaptation skills are represented in the cortico-cerebellar network (Doyon et al., 2002; Doyon, Penhune, \& Ungerleider, 2003). In 2005, Doyon \& Benali re-assessed the literature and proposed that the hippocampus and frontal association areas were also involved in fast learning of both motor sequence learning and motor adaptation. More recently, Penhune and Steele (2012) proposed a model stating that instead of activity in one region indicating fast or slow learning, the contribution of each loop to the phase of motor learning depends on the motor task. For example, motor sequences with a higher cognitive load rely on frontal cortical areas and striatal input. In sum, motor learning relies on many cortical and subcortical structures that summate to form a neural network of motor learning. The activity of each structure and importantly the interaction between these structures varies depending on the task and phase of learning. 
2.3.5 Neural markers of motor learning. Associated with the fast and slow learning phases are changes in brain activity with each stage. The underlying mechanisms of these changes in brain activity likely involve LTP processes occurring in various brain regions across the sensorimotor networks.

During a single motor training session, there is increased functional connectivity between many sensorimotor and cognitive areas including: M1-PMC-SMA, as well as PFC-PMC (Sun, Miller, Rao, \& D'Esposito, 2007). This increase in functional connectivity between these regions allows communication from different areas to prepare, plan, and execute a new movement. In addition to increased functional connectivity, there are increases and decreases of activity in different brain areas across the sensorimotor network. When first introduced to a task there are increases in the blood-oxygen-level dependent (BOLD) activity of cognitive and motor areas (such as M1) relative to rest. Within one session, as the skill is practiced over a short period of time, this BOLD signal increases in the PMC, SMA, parietal areas, basal ganglia, and cerebellum, and decreases in the PFC, M1, and preSMA (Dayan \& Cohen, 2011). The BOLD signal, assessed using functional magnetic resonance imaging (fMRI), is not a direct measure of brain activity however, it has been correlated with activity from postsynaptic potentials (Logothetis, 2008). EEG studies, which also capture activity from postsynaptic potentials, have found increases in brain activity during a single session of motor training in motor preparatory areas. This is represented as an increase in amplitude of the MRPs, specifically, the early component of the MRP which is related to the preparation and planning of movement (Smith \& Staines, 2006, 2010, 2012). These increases in amplitude indicate an increased excitability, which is likely a result of the combination of a reduction in GABA-mediated intracortical inhibition and increase in EPSPs. This combination of disinhibition and excitation makes the 
induction of LTP more likely to occur as it brings the membrane potential closer to the threshold for action potentials to occur. Previous research has hypothesized the disinhibition of intracortical connections as one of the mechanisms underlying the behavioural fast learning phase (Karni et al., 1998). In support of this, Floyer-Lea, Wylezinska, Kincses, and Matthews (2006) found that within a 30 minute motor training session the concentration levels of GABA decreased in the contralateral M1 and S1. This reduction of GABA concentration was accompanied with improvements in performance across the single training session. It is likely that this mechanism assists with the encoding of the motor memory during fast learning, but not the consolidation of the motor memory associated with the transition to slow learning because the GABA levels start to return to baseline soon after the motor training session.

With practice over multiple training sessions, the movement is sequenced together and the representation of the movement in M1 expands to cover more cortical space, paralleling with the behavioural slow learning phase (Karni et al., 1998; Nudo et al., 1996; Nudo, 2003). When this occurs, the individual is able to perform the task with little input from the preparatory and planning regions such as the SMA (Aizawa, Inase, Mushiake, Shima, \& Tanji, 1991) and the cerebellum (Doyon et al., 2002; Galea et al., 2011). Instead, there is a shift to more posterior areas of the cortex that are activated during the motor task such as M1 and S1, and a subcortical area, the dorsolateral striatum (Dayan \& Cohen, 2011). BOLD activity of M1 is increased relative to when the skill was first being learned as a result of the expansion of the cortical representation (Karni et al., 1995, 1998). The relative decrease in brain activity from cognitive and motor preparatory areas is the basis behind the neural efficiency theory which postulates trained individuals (experts) are able to successfully complete the task using the least amount of energy. There is evidence for this occurring in fMRI (Jäncke, Shah, \& Peters, 2000) and EEG 
studies (Wright et al., 2011). In EEG studies this is represented as a decrease in amplitude and shorter latency of MRPs (Wright et al., 2011). The decrease in amplitude of MRPs suggests less synaptic activity is required to produce the motor skill, and the shorter latency of MRPs suggests that less time is needed to plan and prepare the motor skill. Decreases in the amplitude of the MRPs are specifically seen in the electrodes overlaying the SMA and somatosensory areas, suggesting that less planning is needed for these movements (Del Percio et al., 2008). Evidence for neural efficiency has also been found in EEG studies using brain wave analysis. Specifically, experts had lower amplitudes of low- and high-frequency alpha ERD (Del Percio et al., 2010). Such changes in the characteristics of MRPs and changes in alpha waves would likely not occur over a single training session, but rather over the long-term training of the motor skill. However, some studies have found decreases in the amplitude of MRPs during a single motor training session. One study summarized by Wright et al. (2011) found that during the first half of the training session the amplitude of the BP increased, which correlated with improvements in the speed of the button-pressing task (Taylor, 1978). Once the performance plateaued, the BP amplitude did not continue to increase but stayed constant or decreased in amplitude. This was interpreted as an improvement in neural efficiency once the individual had achieved a high performance level. One possibility for neural efficiency to occur within one training session is that the task may be fairly simple and easy to learn.

2.3.6 Bimanual motor training (BMT). Many of the studies referenced above have employed unimanual tasks such as reaching and grasping, or sequences of finger movements and have found that unimanual motor training can induce changes in M1 (ex: Karni et al., 1998). Previous motor learning studies in our research group have employed BMT tasks. BMT is a more complex motor training protocol that uses both upper limbs simultaneously. There are two 
types of movements associated with BMT: inphase movement, when both wrists move in flexion or in extension, and antiphase movement, when the wrists complete opposite movements. BMT tasks are of interest because bilateral movements have been found to increase activity in the afflicted hemisphere of stroke patients (Staines, McIlroy, Graham, \& Black, 2001). Thus, BMT may be beneficial in clinical settings. BMT leads to increases in the amplitude of the early component of the cued MRP, a modulation that is related to a behavioural decrease in response time (RT) and increase in successful trials (Smith \& Staines, 2006). In the context of these studies, only inphase BMT (not antiphase or unimanual training) caused this increase in amplitude in the early MRP (Smith \& Staines, 2010). Similarly, only after inphase BMT does the spatial representation of the extensor carpi radialis (ECR) muscle in M1 expand (Neva, Legon, \& Staines, 2012). This expansion of M1 after inphase BMT is not a long-term change in M1 that occurs over many training sessions (Karni et al., 1998; Nudo et al., 1996; Nudo, 2003) but rather, it is a short-term change that is likely reflective of early LTP-like mechanisms and a disinhibition of already present intracortical connections (Jacobs \& Donoghue, 1991; Stinear \& Byblow, 2002). All this considered, inphase BMT is an effective way to induce short-term changes in excitability, specifically in preparatory motor areas, which could induce LTP processes.

There is some evidence to suggest that the combination of BMT and TMS techniques, such as intermittent theta burst stimulation (iTBS), have additive effects leading to increased amplitude and spatial extent of the motor evoked potential (MEP) (Neva, Singh, Vesia, \& Staines, 2014). In-phase BMT also may reduce the interhemispheric inhibition between the right M1 and left M1, and continuous TBS (cTBS) to the right M1 could increase the excitatory connections between the hemispheres, leading to enhanced cortical excitability (Neva, Vesia, Singh, \& Staines, 2014). This suggests that other interventions that also affect cortical 
excitability, such as aerobic exercise, may be used in combination with BMT to complement its' effects. Although the BMT and TMS interventions above did not investigate the effects on the consolidation of motor memories and retention of the motor skill, the prolonged increase in the ECR spatial map suggests that it may have assisted with consolidation mechanisms.

\subsection{Exercise}

2.4.1 Acute and chronic effects of exercise. It is well-known that aerobic exercise has many benefits for the brain (Hillman, Erickson, \& Kramer, 2008). Chronic exercise leads to more long-term changes such as increases in brain volume (Erickson et al., 2011; Hillman et al., 2008), and acute exercise causes more short-term changes such as increases in cortical excitability (Singh \& Staines, 2015). The effects of acute and chronic exercise are not unrelated as they rely on similar mechanisms, with chronic exercise leading to a cumulative effect of such mechanisms (Roig, Nordbrandt, Geertsen, \& Nielsen, 2013). For example after acute exercise peripheral BDNF levels increase (Skriver et al., 2014; Winter et al., 2007), and after long-term exercise interventions, resting peripheral BDNF levels increase (Huang, Larsen, Ried-Larsen, Møller, \& Andersen, 2014). As well, acute and chronic exercise can lead to increased M1 excitability (Cirillo, Lavender, Ridding, \& Semmler, 2009; Singh, Neva, et al., 2014; Singh \& Staines, 2015). The molecular processes of acute and chronic exercise can interact and complement one another. Studies have shown that exercise-trained animals have an enhanced (hippocampal) BDNF response to acute exercise (Berchtold, Chinn, Chou, Kesslak, \& Cotman, 2005) and individuals with high physical activity levels have a larger cortical excitability response to acute exercise (Lulic, El-Sayes, Fassett, \& Nelson, 2017).

Behaviourally, acute and chronic exercise also lead to similar effects. For example, both acute and chronic exercise can improve short and long-term cognitive function (Kramer \& 
Erickson, 2007; Tomporowski, 2003). However, some aspects of cognition are differently affected by chronic and acute exercise. Acute exercise has a positive effect on short and longterm memory, while chronic exercise has slight improvements on short-term memory and no effect on long-term memory (Roig et al., 2013). Broadly, both chronic and acute exercise can enhance motor skill acquisition and retention (Etnier, Romero, \& Traustadottir, 2001; Quaney et al., 2009; Roig et al., 2012; Statton et al., 2015). While chronic exercise seems to have general enhancement effects, the effects of acute exercise on motor learning depend on when the exercise takes place relative to the motor learning (Roig et al., 2016). In summary, acute and chronic exercise both benefit brain function. Unsurprisingly, the combination of both chronic and acute exercise sessions seems to yield the most benefits to not just motor learning, but many brain functions. The next sections discuss acute exercise more specifically since the current study investigated the effects of acute exercise on the consolidation of motor memories.

2.4.2 The effects of acute exercise on cortical plasticity. Using TMS, researchers have examined how acute exercise can modulate the excitability of the cortex. Singh, Duncan, et al. (2014) had participants complete moderate intensity cycling (65-70\% maximal heart rate (HR)) and compared activity in M1 before and after exercise. TMS was applied to the ECR motor hotspot and was used to measure input-output curves, SICI, long-interval intracortical inhibition (LICI), and intracortical facilitation (ICF). While no differences for the input-output curves or LICI were observed, after exercise there were increases in ICF and decreases in SICI. SICI and LICI are thought to be mediated by different types of GABA receptors (GABAA and GABAB respectively), which may explain why one decreased significantly and the other, although it follows a similar trend, did not. ICF is mediated by glutamate and NMDA receptors. Increases in ICF and decreases in SICI typically need to occur prior to LTP. Thus, acute exercise is thought 
to prime M1 for LTP-like plasticity in this way. Smith, Goldsworthy, Garside, Wood, and Ridding, (2014) also found this decrease in SICI 15 minutes after both low-moderate intensity exercise (40\% predicted HRR), and moderate-high intensity exercise (80\% predicted HRR). This suggests that exercise promotes future cortical modulations by reducing intracortical inhibition. When LTP-like plasticity is experimentally induced through paired associative stimulation (PAS) after moderate intensity exercise, there are greater increases in M1 excitability than when PAS is preceded by a period of rest (Singh, Neva, et al., 2014). This is also accompanied by a reduction in SICI, suggesting that acute moderate intensity exercise selectively reduces GABA A-mediated inhibition to prime the brain for LTP. This same priming effect of moderate intensity exercise is not observed in LTD-like plasticity (McDonnell et al., 2013; Singh, Duncan, \& Staines, 2016). When LTD-like plasticity is experimentally induced through cTBS after moderate intensity exercise, no reduction in MEP amplitude occurs (McDonnell et al., 2013; Singh, Duncan, et al., 2016). Therefore, moderate intensity exercise does not prime LTD-like plasticity, it opposes the induction of LTD-like plasticity, making it less likely to occur. This could be the result of glutamatergic activity demonstrated through increased ICF, or exercise-related increases in LTPrelated compounds which typically oppose LTD.

Exercise does not selectively alter M1 cortical excitability. Other areas in the sensorimotor network are affected and contribute to this change. Results from Thacker et al. (2014) suggest that exercise enhances activity in the motor planning regions of the sensorimotor network, specifically the SMA. Using EEG, Thacker et al. (2014) examined how exercise modulated components of the BP. Participants completed ballistic wrist extension movements before and at two time points after a session of moderate intensity exercise. They found that exercise resulted in an earlier onset of the early BP component at the post measure when HR 
returned to baseline compared to the pre-exercise measure. This finding suggests that exercise affects the motor planning regions of the sensorimotor network, and further that these exerciseinduced modulations are not an arousal effect since HR had returned to baseline when these changes were observed. The cerebellum is another structure in the sensorimotor network that is modulated post-exercise. Similar to the post-exercise reduction in inhibition in M1 observed in the above studies, Mang et al. (2016) found a decrease in cerebellar inhibition post high intensity exercise which enhanced MEP amplitudes. Evidence also suggests that the functional connectivity of resting-state sensorimotor networks are affected by exercise (Rajab et al., 2014). Using fMRI, researchers examined an increase in resting-state functional connectivity between $\mathrm{M} 1, \mathrm{~S} 1$, secondary somatosensory areas, and the thalamus after a session of moderate intensity exercise.

\subsubsection{The effects of acute exercise on motor learning and retention. The role of} exercise is to not to cause LTP, but to prime LTP, increasing the potential for it to occur. Behavioural studies have shown that moderate intensity exercise performed prior to motor training can enhance the acquisition but not the retention of a motor skill (Snow et al., 2016; Statton et al., 2015). This is true for different types of motor tasks, as well as if the exercise intensity is prescribed based on HR (Statton et al., 2015) or peak oxygen $\left(\mathrm{O}_{2}\right)$ uptake (Snow et al., 2016). Thus, pre-motor training exercise primes neural mechanisms responsible for encoding the motor memory. When exercise is performed after motor training, improvements in the retention of motor skills are observed which suggests that post-motor training exercise primes consolidation mechanisms (Roig et al., 2012; Thomas, Flindtgaard, et al., 2016; Thomas, Beck, et al., 2016; Thomas, Johnsen, et al., 2016). Pre-motor training high intensity exercise can also assist with consolidation in addition to enhancing acquisition (Mang, Snow, Wadden, Campbell, 
\& Boyd, 2016; Mang, Snow, Campbell, Ross, \& Boyd, 2014; Stavrinos \& Coxon, 2017; Winter et al., 2007). In this case it affects retention as well as acquisition likely because high intensity exercise has longer-lasting effects that temporally overlap with consolidation mechanisms.

One particular study that has provided insight into this relationship regarding the timing of exercise and motor learning was conducted by Roig et al. (2012). Researchers compared whether acute high intensity exercise influenced motor acquisition and retention, and also whether the timing of exercise changed this relationship. Participants were divided into groups that completed only motor training (control), exercise prior to motor training (PRE), or exercise post-motor training (POST). Exercise was prescribed based on graded exercise tests. The motor task required participants to track a sine wave curve on a computer screen by flexing and extending their wrist to move a single handle which corresponded with vertical cursor movement. One baseline test and three retention tests were performed (1 hour, 24 hours, and 7 days post). Results revealed that both exercise groups had better accuracy in the 24 hour and 7 day retention tests, but the POST group had a better performance in the 7 day retention test compared to the PRE group. These results suggest that aerobic exercise performed after motor training may enhance motor memory consolidation.

The timing and intensity of the exercise can affect retention of the motor skill, but the type of exercise does not (Thomas et al., 2016; Thomas, Beck, et al., 2016; Thomas, Johnsen, et al., 2016). Exercise performed in close temporal proximity to motor acquisition of a unimanual visuomotor tracing task has a better effect on retention performance than exercise completed with a large temporal delay from motor acquisition (Thomas, Beck, et al., 2016). This suggests that there is an optimal temporal window with which exercise post-motor training can interact with consolidation mechanisms. Exercise performed at higher intensities (90\% maximal power 
output) leads to better performance on retention than exercise performed at low intensities (45\% maximal power output) (Thomas, Johnsen, et al., 2016). However, low intensity exercise can still provide some retention benefit over no exercise. Thus, there may be a dose-response relationship between exercise intensity and retention scores. This relationship between exercise intensity and retention performance needs to be further explored to inform motor training protocols.

Exercising at a moderate or low intensity may still be beneficial for populations who cannot participate in high intensity exercise, such as neurorehabilitation patients. The type of exercise does not seem to affect motor memory consolidation as strength training, circuit training, and indoor floor hockey all result in improved retention scores one day later, with no significant differences between them (Thomas et al., 2016). However, participants did not complete a 7 day retention test, therefore it is unknown whether the type of exercise would have different effects at a longer-term retention.

\subsubsection{The correlation of neural markers and behavioural changes. The research}

outlined above leads to questions about the mechanisms behind exercise-related improvements in behaviour and the relationship between such neural and behavioural outcomes. Few studies have investigated the relationship between the neural modulations and behavioural changes associated with the combination of exercise and motor learning (Dal Maso, Desormeau, Boudrias, \& Roig, 2018; Mang et al., 2014; Singh, Neva, \& Staines, 2016; Stavrinos \& Coxon, 2017). The findings of these studies have not shown conclusive correlations, possibly due to a small sample size that is not able to capture the effects. Despite this, there are some significant findings and correlations that informed the current study. Singh, Neva, et al. (2016) found that exercise performed before motor training increased cortical excitability more so than training or exercise alone. However, no behavioural improvements in the motor task were observed. Instead, their results 
demonstrated that exercise and motor training alone both contribute to excitability changes in the cortex. The effects of exercise are more global in M1, and the effects of training are specific to the involved muscles. Specifically, motor training alone led to a significant increase in the number of active sites of the ECR representation, while exercise alone did not. However, the combination of exercise and training significantly increased the central zone MEP amplitude. Mang, Snow, Campbell, Ross and Boyd (2014) did not find any correlations between exerciseenhanced responses to PAS and exercise-related improvements in motor retention. Additionally, increases in peripheral BDNF did not correlate with enhanced responses to PAS or behavioural improvements. Similarly, even though exercise significantly modified neural and behavioural measures, Stavrinos and Coxon (2017) did not find a correlation between exercise-related reductions of SICI and offline improvements. However, when all participants (exercise and control groups) were considered together, they did find a significant correlation between changes in SICI and offline learning. This suggests that a larger sample size is necessary to be able to observe this correlation. Nevertheless, there has been one recent study that has observed a correlation between neural modulations and behavioural improvements (Dal Maso et al., 2018). This study stemmed from the previous behavioural studies of Roig and colleagues as it investigated the cortical modulations underlying improvements in retention associated with postmotor training exercise. EEG was used to assess modulations in alpha and beta ERD, functional connectivity, and CMC. The motor training consisted of a unimanual motor task that required participants to use grip force to move a cursor up and down on the screen to reach 12 targets. They replicated their past behavioural results showing that the exercise group had a better retention of the skill 24 hours later. The exercise group also had increased functional connectivity between sensorimotor areas, some increased beta-band CMC, and a decrease in 
beta-band ERD in the contralateral sensorimotor area. However, the only neural modulation that correlated with enhanced retention was the decrease in beta-band ERD. Beta-band ERD is thought to represent neural activity related to planning and execution of movements. Thus, a decrease in beta-band ERD suggests that exercise assists motor learning by making the neural networks more efficient. That is, less neural activity is needed to perform the task. Beta band ERD, like SICI, is mediated by GABA receptors. Thus, this supports the idea that exercise can suppress inhibition which may prime the cortex for LTP-like plasticity.

In sum, high intensity post-motor training exercise provides a benefit to the retention of the motor skill. While the neural markers of this behavioural improvement have yet to be determined, it is likely that the neurophysiological mechanisms of exercise are the same regardless of the timing of exercise relative to motor training. To further investigate the effect of post-motor training exercise on the consolidation of motor memories and inform motor learning paradigms the next steps should include manipulating motor training and exercise variables, identifying neural markers, and investigating correlations to better understand the mechanisms behind the behavioural changes. 


\subsection{Rationale, Objectives, and Hypotheses}

\subsection{Rationale}

Previous research has suggested that acute aerobic exercise may assist in the consolidation of motor memories (Roig, Skriver, Lundbye-Jensen, Kiens, \& Nielsen, 2012; Thomas et al., 2016; Thomas, Beck, et al., 2016; Thomas, Johnsen, et al., 2016). The timing of exercise relative to motor training seems to play a role in this relationship as studies have found that the long-term retention of the motor skill is improved when exercise is performed immediately after motor training (Lundbye-Jensen, Skriver, Nielsen, \& Roig, 2017; Roig et al., 2016, 2012; Thomas, Beck, et al., 2016). This suggests that exercise performed post-motor training may improve retention scores by priming consolidation mechanisms. The intensity of exercise is also related to the long-term retention of the motor skill as it has been shown that high intensity interval training leads to superior retention scores compared to low intensity interval training (Thomas, Johnsen, et al., 2016). The effects of a continuous moderate intensity exercise have not yet been examined. This study investigated the effects of acute continuous moderate intensity exercise on the consolidation of motor memories. Since there is limited research that has investigated the cortical modulations underlying the behavioural changes of this relationship this study also examined neurophysiological modulations by measuring MRPs. Initial correlational studies suggested some associations between behavioural performance and betaband ERD (Dal Maso et al., 2018). Further electrophysiological research is needed in healthy young adults to investigate how aerobic exercise can assist motor memory consolidation. These results inform motor learning paradigms and future studies with other populations including older adults and neurorehabilitation patients. 


\subsection{Objectives \& Hypotheses}

The objectives of the study were as follows:

1. Examine whether a session of continuous moderate intensity aerobic exercise performed after motor training would affect the consolidation of the motor skill as measured by performance at retention measures.

Main hypothesis: EXE would have a significantly lower change in RMS and RT scores compared to $\mathrm{CON}$ from the last 5 trials of post-training 2 to the retention measures. This was predicted from previous behavioural results (Kantak et al., 2010, 2011; Lundbye-Jensen et al., 2017; Roig et al., 2012).

In addition to our main hypothesis we assumed that the motor training would improve RMS and RT scores. Therefore, we predicted that both EXE and CON would have significant improvements in RMS and RT at late training and post-training measures and that at these time points there would be no significant difference between the groups. Past research has indicated that performance differences between groups do not emerge immediately after the intervention (Lundbye-Jensen et al., 2017; Roig et al., 2012).

2. Investigate the influence of motor training and aerobic exercise on cortical activity relating to motor preparation and execution (MRPs).

Main hypothesis: EXE would have a larger amplitude increase of the early MRP component at the post-training measure compared to CON. This was predicted from recent research that suggests there are changes in motor preparatory regions post-exercise (Dal Maso et al., 2018).

In addition to our main hypothesis we predicted that both groups would have a larger amplitude of the early MRP component at the late training and post-training measure compared 
to the early training measure. This was based on studies that have demonstrated that inphase BMT alone causes an increase in the amplitude of the early component (Smith \& Staines, 2010; Smith \& Staines, 2006).

3. Investigate the relationship between behavioural measures and MRP changes.

Hypothesis: There would be a correlation between increases in the amplitude of the early MRP component and changes in the RMS and RT score from the last 5 trials of post-training 2 to retention. This hypothesis was based on recent findings that demonstrate a correlation between cortical activity in motor preparatory areas and motor skill retention (Dal Maso et al., 2018). 


\subsection{Methods}

\subsection{Participants.}

Participants were healthy young adults between the ages of 18-35, recruited from the University of Waterloo community. There were 34 participants recruited to participate in the study and 33 participants included in the study with 17 participants in EXE and 16 participants in CON. Exclusion criteria assessed by the University of Waterloo Health History Questionnaire consisted of the following: injury that makes exercise uncomfortable, any medications affecting the central nervous system, history of seizure/epilepsy, any neurological injury/disease, and recent history of concussion. Additionally, any individual accumulating less than 600 metabolic equivalents (METs) per week as assessed by the International Physical Activity Questionnaire (IPAQ) was excluded from the study. The Get Active Questionnaire (GAQ) was used to determine if the individual could safely participate in exercise. Informed written consent was obtained before beginning the study and participants were allowed to withdraw from the study at any point for any reason. Once the data was collected, individual identifiers were removed from the data to ensure participant anonymity. Upon completion of the study participants were compensated for their time.

\subsection{Procedures.}

Prior to collection, participants completed the Positive and Negative Affect Schedule (PANAS) scale to assess their current affect, the Stanford Sleepiness Scale (SSS) to determine their current level of sleepiness, and the Edinburgh Handedness Questionnaire (EHQ) to evaluate handedness. Affect and sleepiness may contribute to arousal levels which could influence learning and performance of the motor task. Handedness is not likely to affect the task since it is bimanual, however it would provide some explanation should one hand be dominant in the task. 
Participants were also asked to provide an estimate of their weight and height so body mass index (BMI) could be calculated. Once completed, participants were randomly assigned to one of two groups: CON or EXE. Participants were given instructions for the task and were oriented and familiarized with the custom-made BMT device used previously in our lab (Neva, Singh, Vesia, \& Staines, 2014; Singh et al., 2016; Smith \& Staines, 2006, 2010, 2012). After this, as illustrated in Figure 1, participants performed the baseline test and then completed the motor training. The CON group performed the motor training and then rested for a period of 30 minutes. During the rest period they read while they were in a seated position on the cycle ergometer. After motor training the EXE group performed a session of aerobic exercise for 30 minutes. Both groups then completed a second PANAS scale to assess any mood differences post-exercise or rest. Two post-training tests were completed, one 10 minutes after the rest/exercise session (post-training test 1), and one once HR had returned to baseline in the EXE group and 30 minutes after rest in the CON group (post-training test 2) (Singh, Duncan, et al., 2014; Singh, Neva, et al., 2016). This second post-training test was to account for fatigue and arousal effects that may have affected performance in the first post-training test (Roig et al., 2016). During the time between the post-training test 1 and post-training test 2 both groups were provided with reading material. Participants returned to the lab 1 day and 7 days later to complete retention and transfer tests. Two participants returned 2 days and 8 days later due to campus closure. Prior to completing the retention and transfer tests participants completed the PANAS scale and the SSS. On visit 2 they also completed the St. Mary's Hospital Sleep Questionnaire (SMHSQ). The SMHSQ provided an indication of the quality and quantity of sleep the night after the experimental session. Sleep is important to measure as it can affect the consolidation of motor memories (King et al., 2017). All three visits for each participant were 
completed around the same time of day (within 3 hours). Consistent with past studies, participants were required to refrain from physical activity 2 hours before each visit and 4 hours after the first visit (Borota et al., 2014; Thomas et al., 2016; Thomas et al., 2016). Participants were instructed to maintain their typical caffeine intake and avoid sleeping for 4 hours after the first visit.

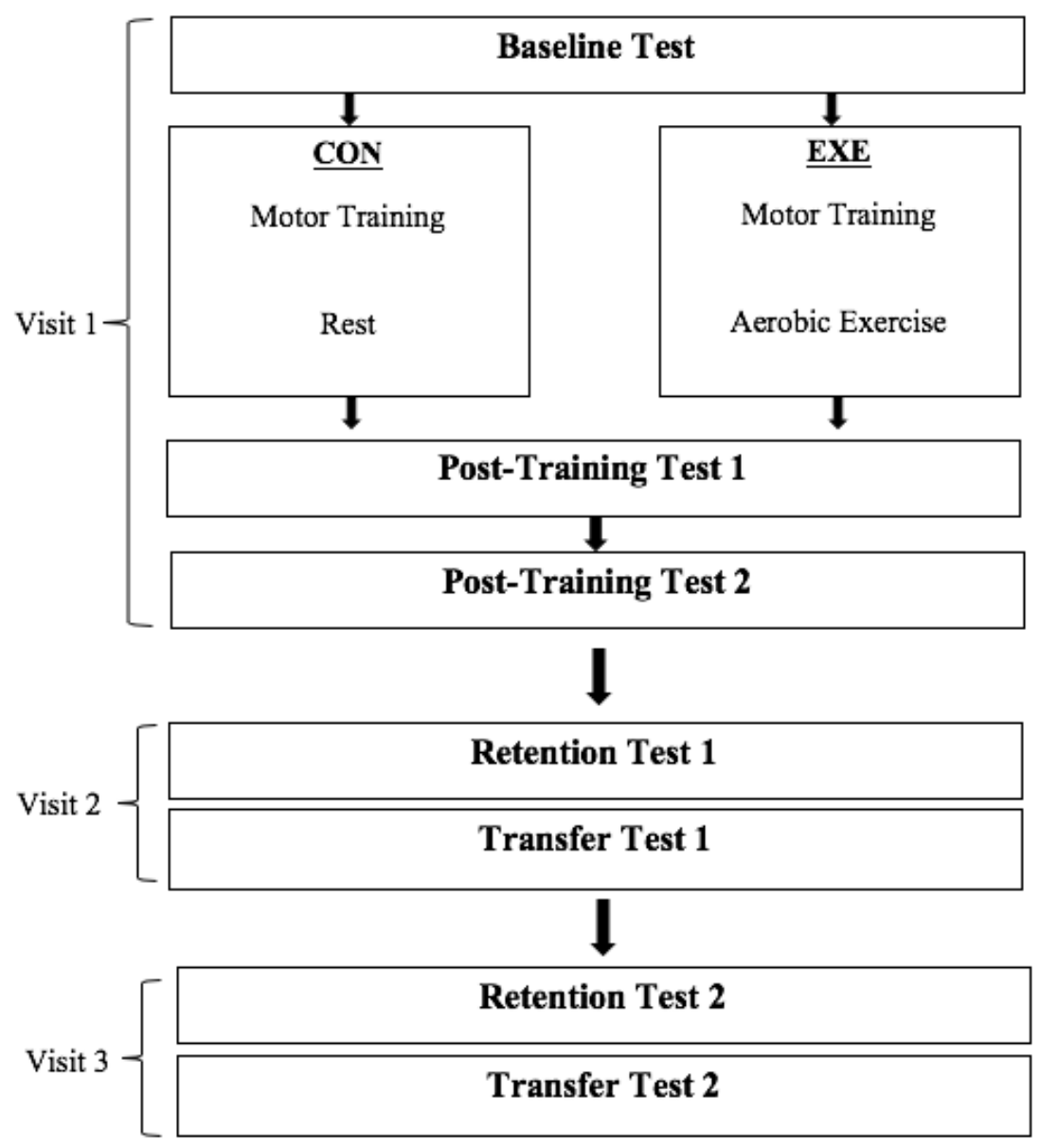

Figure 1. General procedures

\subsection{Exercise.}

Prior to exercise a baseline HR was collected. HR was assessed using a wristwatch-tochest heart rate monitor (Léger \& Thivierge, 1988). Rate of perceived exertion (RPE) was assessed using the Borg scale (Borg, 1970). Target RPE was between 12-15 to reflect a moderate intensity. The exercise was a 20 minute continuous moderate intensity session on a stationary 
recumbent bike. During exercise arms were resting on the handle bars and participants were instructed to keep their arms relaxed to avoid fatiguing the muscles involved in the motor training task. Cycling was chosen based on past literature that has demonstrated that lower body aerobic exercise can modulate excitability in the motor cortical representations for the upper body (Singh, Duncan, et al., 2014). Participants completed a 5-minute self-paced warm-up to get their HR up to a moderate intensity level, which was calculated as $70 \%$ of their HRR based on their age-predicted HR max and resting HR (i.e., 70\% HRR $\left.=0.7\left(H R_{\max }-H R_{\text {rest }}\right)+H R_{\text {rest }}\right)$. Once they reached this HR at the end of the 5 minute warm-up, they exercised at this intensity for 20 minutes. HR was monitored continuously throughout the session and RPE was reported every 5 minutes. Participants kept a pace of 55-65 revolutions per minute (RPM) and adjusted the bike's resistance level to maintain target HR (Singh, Neva, et al., 2016; Thacker et al., 2014). After 20 minutes at this intensity participants completed a 5-minute cool-down after which HR and RPE measures were taken again. The intensity and duration of exercise were selected to be less intense than high intensity interval training and more intense than a low intensity interval or continuous exercise bout. This intensity was meant to challenge participants but not exhaust them. Continuous moderate intensity exercise has previously been shown to induce cortical modulations (Singh et al., 2014, 2016; Thacker et al., 2014). Additionally, no known studies have examined the effects of post-motor training continuous moderate intensity exercise on retention of motor skills. Addressing this gap in the literature is important because high intensity interval training is not appropriate for all populations.

\subsection{Motor Task.}

The motor training task was a modified version of the BMT task that has been validated by past motor learning studies from our lab that have examined behavioural and cortical 
modulations (Neva, Singh, Vesia, \& Staines, 2014; Singh et al., 2016; Smith \& Staines, 2006, 2010, 2012). The details of the seating arrangement, training apparatus, and task specifics are outlined in Singh et al. (2016). A figure of the set-up is illustrated in Figure 2. The task required participants to hold two handles, one in each hand, of the custom-made BMT device. The handles were connected to potentiometers to measure their movement. A customized LabVIEW program allowed for the movement of the handles to correspond to movement of the cursor on a screen. Flexion/extension of the right wrist moved the cursor vertically and flexion/extension of the left wrist moved the cursor horizontally. The subject's view of the screen during the motor training is shown in Figure 3. During a single trial participants first saw the cursor (black circle) and moved it to the start position (x) in the bottom right corner. When this occurred, the cursor disappeared and 1 of 3 possible targets $\left(30^{\circ}, 45^{\circ}\right.$, and $60^{\circ}$ from the y-axis) appeared in the top left corner. After a $2 \mathrm{~s}$ delay the cursor reappeared which was the cue for participants to move the cursor to the specified target location. This ensured the measurement of the early cued-MRP component that starts approximately $2 \mathrm{~s}$ before movement. The participant then had $2 \mathrm{~s}$ to move the cursor to the target. After this feedback was given in the form of RT. This was not a true RT, but rather was calculated as the time between when the trial started and when the cursor reached the target. Participants started the next trial when they were ready. The movement from the start position to the targets required participants to complete inphase BMT as used by Singh et al. (2016) shown in Figure 4. This was specifically selected because of past research that has demonstrated that inphase BMT enhances the amplitude of the cued-MRP early component (Smith and Staines, 2010) and can also increase cortical excitability of M1 (Singh, Neva, et al., 2016) suggesting the beginning of early plasticity processes. Participants were told the objective 
of the task was to flex both wrists simultaneously to move the cursor onto the target and to perform the task as quickly and as accurately as possible within the $2 \mathrm{~s}$ timeframe.

The motor training was a random variable practice structure consistent with previous studies (Kantak et al., 2010, 2011). Participants completed 180 trials of training of which 50\% were the test target $\left(45^{\circ}\right), 25 \%$ were a target located $30^{\circ}$ from the $y$-axis, and the remaining $25 \%$ were a target located $60^{\circ}$ from the y-axis. This equated to 90 trials of the test target, and 45 trials each of the other two targets. The 180 trials were completed in a randomized order. Before training participants completed 5 familiarization trials and 5 baseline trials to the test target. Post-training test 1 and retention test 1 and 2 were identical to the baseline test. Post-training test 2 consisted of a larger amount of trials (50 trials) to the test target to ensure enough EEG data was collected to be able to average an MRP trace. Transfer test 1 and 2 were 5 trials of a new target (approximately $37.5^{\circ}$ and $52.5^{\circ}$ from the y-axis). The transfer test was included as an alternative measure of learning. During the baseline, post-training, retention, and transfer tests no feedback of RT was given.

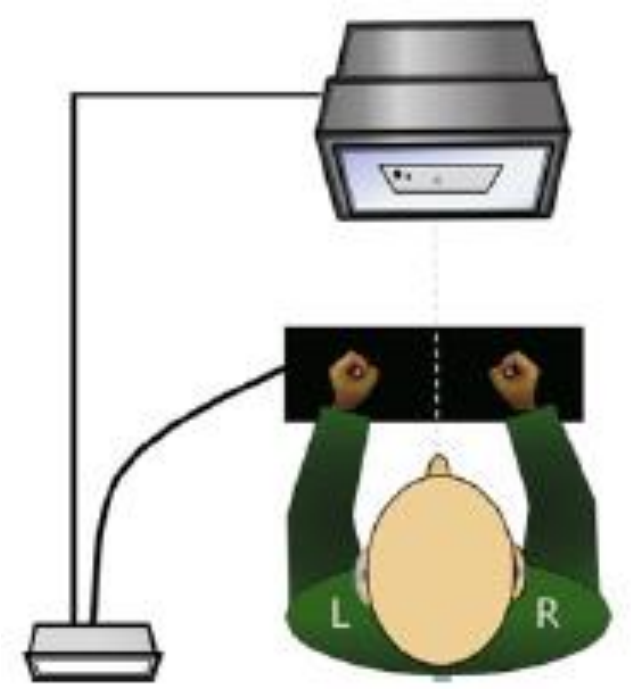

Figure 2. Experimental set-up (Neva et al., 2012) 

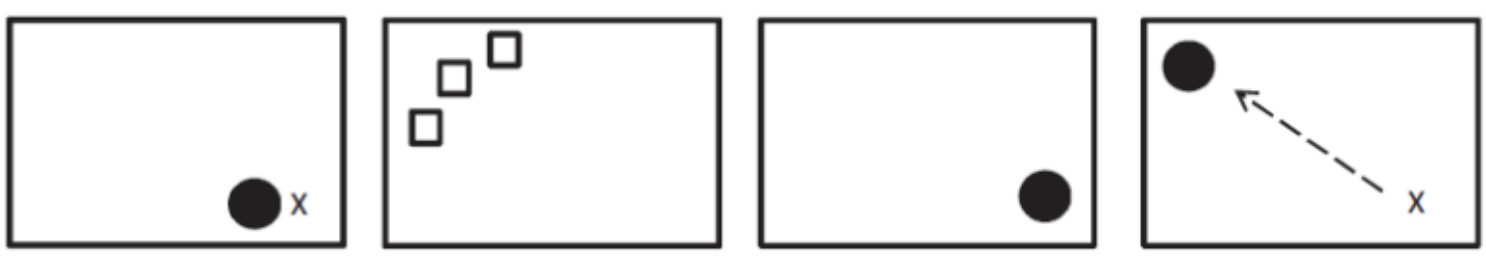

Figure 3. Consecutive view of screen during a single acquisition trial (Singh et al., 2016)
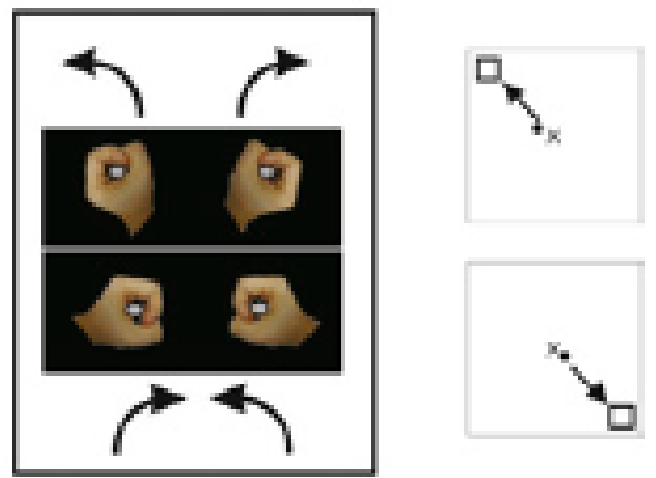

Figure 4. Inphase movement (Neva et al., 2012)

\subsection{Data acquisition.}

EEG was only collected during the training and post-training test 2 at visit 1 . EMG was collected on all days at all timepoints. EMG electrodes were placed on the muscle bellies of the flexor carpi radialis (FCR) of each arm, and the ground electrodes were placed on the clavicles after proper skin preparation according to the SENIAM recommendations (Hermens, Freriks, Disselhorst-Klug, \& Rau, 2000). The EMG data was amplified (x1000), band pass filtered (10$500 \mathrm{~Hz}$ ), and digitized at a rate of $1000 \mathrm{~Hz}$.

EEG was recorded from a 32 channel cap (Quik-Cap, Neuroscan, Compumedics, NC, USA) according to the International 10-20 system. Ten electrodes recorded electrical activity over frontal and sensorimotor areas (FZ, F3, F4, FC3, FCZ, FC4, C3, CZ, C4, PZ). FP1 was also collected to determine blinks. Reference electrodes were placed on the left and right mastoid processes. Impedances of all electrodes were maintained below $5 \mathrm{k} \Omega$. Data was amplified, 
filtered (DC-200 Hz, 6dB octave roll-off) and digitized (1000 Hz, SynAmps2, Scan 4.5, Compumedics Neuroscan, Charlotte, NC) before being stored for off-line analysis.

Behavioural data was collected in the form of RT and the trajectory of the cursor. Event codes from the customized LabVIEW program indicated the start of the trial, when the target appeared, cue to move, start of cursor movement, and the end of the trial. Voltage data from the potentiometers and cursor location were recorded in the customized LabVIEW program. This data was collected at a sample rate of $40 \mathrm{~Hz}$.

\subsection{Data analysis.}

The EEG data was analyzed in Neuroscan (Compumedics Neuroscan, NC, USA)

software. The customized LabVIEW program sent event codes to the continuous EEG file. MRPs were extracted from the EEG data by averaging artifact-free baseline-corrected individual epochs that were time-locked to the onset of cursor movement and extended from $2 \mathrm{~s}$ before to $500 \mathrm{~ms}$ after the onset of cursor movement. Averaged MRP traces representing cortical activity at early training, late training, and post-training 2 were created for each participant. The first 60 trials of training were used to represent early training, and the last 60 trials of training were used to represent late training. All 50 of the post-training 2 trials were used to represent post-training 2. An average of 6 epochs were removed from each average trace due to artifacts. From each averaged MRP trace an area report was used to sum all data points of the trace together from the time window of -1250 to 0 . This time was selected to include cortical activity during planning and execution of the skill. This sum provided a measure of the amplitude of the early MRP. MRP amplitudes were maximal over the midline electrodes so statistical analysis was run on the data from FCZ and CZ. 
To assess accuracy, a customized LabVIEW program compared the actual trajectory of the cursor to the ideal trajectory of the cursor. The program computed an 'ideal' trajectory by creating a straight line from the start position to the target over the time period of $2 \mathrm{~s}$. This 'ideal' trajectory was then compared point-by-point to the actual trajectory resulting in directional error values in the form of pixel co-ordinates. These error values were squared and summed, and the RMS value was calculated as the square root of this sum. Therefore, the magnitude of the RMS value reflects the amplitude of the difference between the actual and the 'ideal' trajectory and the units of the RMS values are arbitrary.

EMG was initially included in the study to be able to study the components of a true RT (i.e., reaction time and movement time). However, since the BMT task is not a true reaction time task due to the predictability of the cue, the EMG data was not analyzed for reaction time, instead time to target was used as a RT measure. This RT was calculated as the time between the cue to move and when the cursor reached the target. If the participant did not reach the target their RT was recorded as $2000 \mathrm{~ms}$ which was the maximum amount of time participants had to reach the target.

For the behavioural data (RMS and RT), at each timepoint (baseline, early training, late training, post-training 1, post-training 2 , retention 1 , retention 2) 5 trials to the test target were averaged to represent performance. The baseline, post-training 1, retention tests all only consisted of 5 trials to the test target. The first 5 trials and the last 5 trials to the test target in the motor training session were averaged to represent early training and late training performance respectively. For post-training 2, the first 5 trials were averaged to represent performance. The last 5 trials of post-training 2 were averaged and used to normalize the retention and transfer 
scores to their performance at the end of visit 1 . For the transfer tests, the 5 trials to the new targets were averaged. 


\subsection{Proposed Hypotheses Testing}

Our main objective was to examine whether a session of continuous moderate intensity aerobic exercise performed after motor training would affect the consolidation of the motor skill as measured by performance at retention measures. To analyze this data a mixed-model Analysis of Variance (ANOVA) for each behavioural measure was used with time (retention 1, retention $2)$ as the within-subjects factor and group $(\mathrm{EXE} / \mathrm{CON})$ as the between-subjects factor. To test our main hypothesis that EXE would have a significantly lower change in RMS and RT scores compared to $\mathrm{CON}$ from the last 5 trials of post-training 2 to the retention measures pre-planned contrasts were used to compare groups at the retention time points. To normalize the RMS and RT scores the last 5 trials of each participant's post-training 2 test was subtracted from their scores at retention 1 , and retention 2 . To test our assumption that there would be significant improvements in RMS and RT at late training and post-training measures and no differences between the groups at these time points, a mixed-model ANOVA for each behavioural measure was used with time (baseline, early training, late training, post-training 1, post-training 2 ) as the within-subjects factor and group (EXE/CON) as the between-subjects factor. Our second objective was to investigate the influence of motor training and aerobic exercise on cortical activity relating to motor preparation and execution. To test our hypothesis that EXE would have a larger amplitude of the early MRP component at the post-training measure compared to CON, and our prediction that both EXE and CON would have a larger amplitude of the early MRP component at the late training measure compared to the early training measure we used a mixedmodel ANOVA. The within-subjects factor was time (early training, late training, and posttraining 2) and the between-subjects factor was group (EXE/CON). Pre-planned contrasts were used to compare early and late time points. For all ANOVA tests and assumptions of normality, 
homogeneity of variance, and sphericity, SAS University Edition was used. To investigate whether there was a relationship between behavioural measures and MRP changes we examined the correlation between change in the amplitude of the early component and change in RMS and RT from the last 5 trials of post-training 2 to retention. Correlational analysis were conducted using the Pearson correlation. A $t$ value was calculated from the coefficient and a two-tailed $t$ test was run to test for significance. 


\subsection{Results}

\subsection{Participant Characteristics}

A total of 34 participants were recruited for the study. Of these 34 participants, one was excluded for accumulating less than 600 METs per week. Therefore, the total number of participants collected was 33 with 17 participants in EXE and 16 participants in CON (Table 1). Of the 33 participants, 19 were female and 14 were male (EXE 10F, 7M; CON 9F, 7M). The age range of participants was $19-26$ and the average age ( \pm standard deviation) was $21.93 \pm 2.13$. The average BMI score in EXE was $24.64 \pm 3.39$ and in CON was $22.76 \pm 2.01$. All participants were deemed safe to participate in exercise from the GAQ. According to the EHQ, 3 participants were lefthanded, one was dominant in an equal number of tasks for both hands, and the rest were righthanded. From the IPAQ a total of 23 participants were categorized as being highly physically active which equates to vigorous-intensity activity on at least 3 days per week and accumulating at least 1500 MET-minutes per week, or 7 or more days of any combination of walking, moderate or vigorous intensity activities accumulating at least 3000 MET-minutes per week. Ten participants were categorized as being moderately physically active which equates to 3 or more days of vigorous intensity activity of at least 20 minutes per day, or 5 or more days of moderate intensity activity and/or walking at least 30 minutes per day, or 5 or more days of any combination of walking, moderate intensity or vigorous intensity activities achieving a minimum of at least 600 MET minutes per week. There were 13 highly active participants in EXE and 10 highly active participants in CON. The average MET-minutes per week was 3017.12 \pm 1627.19 . A two-tailed t test revealed that the average MET-minutes per week in the EXE (3263.05 \pm 1817.26) and in CON (2755.81 \pm 1408.73$)$ were not significantly different $\left(\mathrm{t}_{31}=0.89, p=0.37\right)$. 
Table 1

Participant Characteristics

\begin{tabular}{|c|c|c|c|c|c|c|c|}
\hline Participant & Group & Gender & Age & $\begin{array}{l}\text { IPAQ } \\
\text { Category }\end{array}$ & $\begin{array}{l}\text { MET- } \\
\text { minutes/week }\end{array}$ & Handedness & BMI \\
\hline 1 & EXE & $\mathrm{F}$ & 21 & Moderate & 2412 & $\mathrm{R}$ & 21.8 \\
\hline 2 & EXE & $\mathrm{M}$ & 20 & High & 4980 & $\mathrm{~L}$ & 23.9 \\
\hline 3 & $\mathrm{CON}$ & M & 26 & Moderate & 1065 & $\mathrm{R}$ & 20.5 \\
\hline 4 & $\mathrm{CON}$ & $\mathrm{F}$ & 21 & High & 4709 & $\mathrm{R}$ & 20.1 \\
\hline 5 & EXE & $\mathrm{M}$ & 26 & High & 3147 & $\mathrm{R}$ & 22.4 \\
\hline 6 & $\mathrm{CON}$ & $\mathrm{F}$ & 23 & High & 2121 & $\mathrm{~L}$ & 21.6 \\
\hline 7 & $\mathrm{CON}$ & M & 21 & High & 1710 & $\mathrm{R}$ & 25.8 \\
\hline 8 & EXE & $\mathrm{F}$ & 23 & High & 4990 & $\mathrm{R}$ & 24.3 \\
\hline 9 & EXE & $\mathrm{F}$ & 23 & Moderate & 1048.5 & $\mathrm{R}$ & 22.5 \\
\hline 10 & $\mathrm{CON}$ & $\mathrm{F}$ & 23 & High & 2594 & $\mathrm{R}$ & 23.3 \\
\hline 11 & EXE & $\mathrm{F}$ & 25 & High & 3393 & $\mathrm{R}$ & 21.6 \\
\hline 12 & $\mathrm{CON}$ & M & 23 & Moderate & 1704 & $\mathrm{R}$ & 24.1 \\
\hline 13 & EXE & M & 19 & Moderate & 1012 & $\mathrm{R}$ & 29.3 \\
\hline 14 & $\mathrm{CON}$ & M & 24 & Moderate & 2373 & $\mathrm{R}$ & 26.2 \\
\hline 15 & EXE & M & 19 & High & 2388 & $\mathrm{R}$ & 27 \\
\hline 16 & EXE & $\mathrm{F}$ & 24 & High & 4868 & $\mathrm{R}$ & 21.9 \\
\hline 17 & $\mathrm{CON}$ & $\mathrm{M}$ & 22 & High & 3660 & $\mathrm{R}$ & 20.2 \\
\hline 18 & $\mathrm{CON}$ & $\mathrm{F}$ & 21 & High & 3485 & $\mathrm{R}$ & 22.7 \\
\hline 19 & EXE & $\mathrm{M}$ & 20 & High & 1759 & $\mathrm{~L} / \mathrm{R}$ & 35 \\
\hline 20 & EXE & $\mathrm{F}$ & 20 & High & 2982 & $\mathrm{R}$ & 23.2 \\
\hline 21 & $\mathrm{CON}$ & M & 21 & Moderate & 1400 & $\mathrm{R}$ & 23.7 \\
\hline 22 & EXE & $\mathrm{M}$ & 19 & High & 3772.5 & $\mathrm{R}$ & 24.3 \\
\hline 23 & $\mathrm{CON}$ & $\mathrm{F}$ & 22 & Moderate & 893 & $\mathrm{R}$ & 25.1 \\
\hline 24 & $\mathrm{CON}$ & $\mathrm{F}$ & 20 & High & 3006 & $\mathrm{R}$ & 20.5 \\
\hline 25 & $\mathrm{CON}$ & $\mathrm{F}$ & 21 & High & 2600 & $\mathrm{R}$ & 20.8 \\
\hline 26 & EXE & $\mathrm{F}$ & 19 & High & 2764 & $\mathrm{~L}$ & 23.9 \\
\hline 27 & EXE & $\mathrm{F}$ & 22 & High & 8412 & $\mathrm{R}$ & 26.3 \\
\hline 28 & $\mathrm{CON}$ & $\mathrm{F}$ & 19 & High & 6125 & $\mathrm{R}$ & 22.7 \\
\hline 29 & EXE & $\mathrm{M}$ & 24 & Moderate & 1475 & $\mathrm{R}$ & 23.7 \\
\hline 30 & EXE & $\mathrm{F}$ & 22 & High & 3333 & $\mathrm{R}$ & 22 \\
\hline 31 & CON & $\mathrm{F}$ & 24 & High & 4212 & $\mathrm{R}$ & 24.2 \\
\hline 32 & $\mathrm{CON}$ & $\mathrm{M}$ & 26 & Moderate & 2436 & $\mathrm{R}$ & 22.7 \\
\hline 33 & EXE & $\mathrm{F}$ & 21 & High & 2736 & $\mathrm{R}$ & 25.8 \\
\hline
\end{tabular}

\subsection{Supplementary Data}

Affect scores from the PANAS scale were compared using a mixed-model ANOVA. The within-subjects factor was time (pre-intervention, post-intervention, day 2, day 3 ) and the between-subjects factor was group (EXE/CON). Results revealed a significant interaction effect between group and time $\left(\mathrm{F}_{3,93}=11.88, p<0.01\right)$. Post-hoc comparisons using the Tukey-Kramer test indicated that EXE pre-intervention mean score $(39.97 \pm 3.34)$ was significantly different 
than CON pre-intervention mean score $(37.56 \pm 3.29)$ as well as CON post-intervention $(36.81 \pm$ 3.45), and CON day 2 mean score $(37.81 \pm 3.37)$. EXE also had post-intervention mean score $(41.73 \pm 3.50)$ that was significantly different from all of the CON scores (pre-intervention, postintervention, day 2, day $3: 38.37 \pm 4.35)$ and EXE day $2(38.64 \pm 4.43)$ and day $3(38.20 \pm 3.22)$ scores. The only score that was not significantly different from EXE post-intervention score was EXE pre-intervention score. There were no significant main effects of time $\left(\mathrm{F}_{3,93}=2.28, p=\right.$ $0.08)$ or group $\left(\mathrm{F}_{1,31}=3.06, p=0.09\right)$.

On the SSS all participants rated their degree of sleepiness between a 1-3 except for one participant who during the first visit rated their sleepiness as a $4(1=$ feeling active, vital, alert, or wide awake; 2 = functioning at high levels, but not at peak; able to concentrate; $3=$ awake, but relaxed; responsive but not fully alert; 4 = somewhat foggy, let down). According to the SSS a score above 3 is considered 'sleepy' (Berry \& Wagner, 2014). The mode of all SSS scores was 2. Statistical analyses were not carried out on this data.

Sleep data collected from the SMHSQ was not scored or statistically analyzed but was collected to help explain any outliers. On average participants had $7.25 \pm 1.15$ hours of sleep the night of visit 1. The amount of sleep ranged from 4.6-9.5 hours. All participants rated their sleep as 'fairly well', 'well', or 'very well' except for one participant who rated their sleep as 'fairly badly'.

\subsection{Exercise Data}

EXE had a resting HR of $66.29 \pm 6.19 \mathrm{bpm}$ and CON had a resting HR of $63.75 \pm 9.78$ bpm. The average target HR (70\% of HRR) for EXE was $158.64 \pm 2.37 \mathrm{bpm}$. The average HR for EXE during the 20 minute exercise was $156.01 \pm 3.82 \mathrm{bpm}$. The average HR for CON during the rest session was $65 \pm 9.73 \mathrm{bpm}$. Across all participants in EXE the HRR percentage reached 
during exercise ranged from $62.40-74.81 \%$ with the average HRR percentage at $67.91 \% \pm 3.82$. The average RPE score was $14.13 \pm 1.05$. Participants in EXE maintained an average RPM of $61.01 \pm 2.45$ and all individual averages were within the required range of 55-65. After exercise during the post-training test 1 the average $\mathrm{HR}$ for EXE was $91.82 \pm 7.10 \mathrm{bpm}$ and for CON was $63.88 \pm 8.80 \mathrm{bpm}$. For post-training test 2 , it took EXE an average of $30.35 \pm 10.71$ minutes for HR to return to near resting levels. Due to time constraints one participant completed posttraining test 2 before their HR was back to resting levels (indicated by the * in Table 2). CON completed post-training test 2 an average of $30.25 \pm 1$ minutes after the rest session. At posttraining test 2 the average HR for EXE was $71.47 \pm 7.68 \mathrm{bpm}$ and for CON was $65.29 \pm 10.31$ bpm. 
Table 2

HR, RPE, and RPM

\begin{tabular}{|c|c|c|c|c|c|c|c|c|c|c|}
\hline Participant & Group & $\begin{array}{l}\text { Rest } \\
\text { HR }\end{array}$ & $\begin{array}{l}\text { Target } \\
\text { HR }\end{array}$ & $\begin{array}{l}\text { Exercise/Rest } \\
\text { Session AVG } \\
\text { HR }\end{array}$ & $\begin{array}{l}\text { AVG \% of } \\
\text { HRR during } \\
\text { Exercise }\end{array}$ & $\begin{array}{l}\text { AVG } \\
\text { RPE }\end{array}$ & $\begin{array}{l}\text { AVG } \\
\text { RPM }\end{array}$ & $\begin{array}{l}\text { HR } \\
\text { Post1 }\end{array}$ & $\begin{array}{l}\text { HR } \\
\text { Post2 }\end{array}$ & $\begin{array}{l}\text { Rest/exercise } \\
\text { to Post2 } \\
\text { (min) }\end{array}$ \\
\hline 1 & EXE & 69 & 160 & 158.5 & 68.85 & 13.5 & 59 & 93 & 70 & 50 \\
\hline 2 & EXE & 62 & 158 & 154.75 & 67.21 & 15.25 & 62.75 & 93 & 68 & 38 \\
\hline 3 & $\mathrm{CON}$ & 62 & 154 & 63 & - & - & - & 60 & 64 & 30 \\
\hline 4 & $\mathrm{CON}$ & 52 & 155 & 52.5 & - & - & - & 51 & 50 & 30 \\
\hline 5 & EXE & 62 & 154 & 150.5 & 67.045 & 16 & 61.25 & 91 & 64 & 51 \\
\hline 6 & $\mathrm{CON}$ & 66 & 158 & 66.5 & - & - & - & 62 & 67 & 30 \\
\hline 7 & $\mathrm{CON}$ & 53 & 155 & 55.5 & - & - & - & 55 & 53 & 30 \\
\hline 8 & EXE & 73 & 160 & 153.25 & 64.72 & 13 & 56.75 & 94 & 78 & 19 \\
\hline 9 & EXE & 74 & 160 & 154.75 & 65.65 & 14 & 57.5 & 89 & 78 & 21 \\
\hline 10 & $\mathrm{CON}$ & 63 & 157 & 67.75 & - & - & - & 63 & 65 & 30 \\
\hline 11 & EXE & 74 & 159 & 163 & 73.55 & 13.125 & 62.25 & 88 & 79 & 18 \\
\hline 12 & $\mathrm{CON}$ & 56 & 155 & 58.75 & - & - & - & 61 & 61 & 30 \\
\hline 13 & EXE & 66 & 160 & 154 & 65.19 & 15 & 63 & 87 & 68 & 38 \\
\hline 14 & CON & 65 & 157 & 59.75 & - & - & - & 67 & 62 & 34 \\
\hline 15 & EXE & 70 & 162 & 160.5 & 69.08 & 14.75 & 62.25 & 105 & 75 & 29 \\
\hline 16 & EXE & 60 & 155 & 161.75 & 74.82 & 12.75 & 61.25 & 97 & 66 & 31 \\
\hline 17 & $\mathrm{CON}$ & 52 & 154 & 53.5 & - & - & - & 53 & 52 & 30 \\
\hline 18 & $\mathrm{CON}$ & 54 & 155 & 57.25 & - & - & - & 58 & 58 & 30 \\
\hline 19 & EXE & 67 & 160 & 150 & 62.41 & 14.75 & 62.25 & 93 & 72 & 18 \\
\hline 20 & EXE & 70 & 161 & 158 & 67.69 & 15.125 & 57.25 & 93 & 74 & 30 \\
\hline 21 & $\mathrm{CON}$ & 74 & 161 & 76.5 & - & - & - & 69 & 74 & 30 \\
\hline 22 & EXE & 66 & 160 & 158.75 & 68.70 & 11.75 & 59.75 & 102 & $88^{*}$ & 31 \\
\hline 23 & $\mathrm{CON}$ & 68 & 159 & 73.5 & - & - & - & 69 & 72 & 30 \\
\hline 24 & $\mathrm{CON}$ & 72 & 162 & 75.5 & - & - & - & 66 & 71 & 30 \\
\hline 25 & $\mathrm{CON}$ & 88 & 166 & 86.75 & - & - & - & 89 & 88 & 30 \\
\hline 26 & EXE & 62 & 159 & 153.75 & 66.01 & 14.5 & 64.75 & 89 & 62 & 26 \\
\hline 27 & EXE & 72 & 160 & 156 & 66.67 & 14 & 62.25 & 94 & 77 & 27 \\
\hline 28 & $\mathrm{CON}$ & 73 & 163 & 73.75 & - & - & - & 67 & 73 & 30 \\
\hline 29 & EXE & 70 & 158 & 158.5 & 70.24 & 14.25 & 63.25 & 91 & 75 & 35 \\
\hline 30 & EXE & 52 & 154 & 151.5 & 68.15 & 14.625 & 58.25 & 71 & 57 & 19 \\
\hline 31 & $\mathrm{CON}$ & 61 & 154 & 67 & - & - & - & 59 & 62 & 30 \\
\hline 32 & $\mathrm{CON}$ & 61 & 154 & 59 & - & - & - & 64 & 57 & 30 \\
\hline 33 & EXE & 58 & 157 & 154.75 & 68.62 & 14 & 63.5 & 91 & 64 & 35 \\
\hline
\end{tabular}




\subsection{Behavioural Data}

A mixed-model ANOVA on the RMS retention data (Figure 5) with time (retention 1, retention 2) as the within-subjects factor and group (EXE/CON) as the between-subjects factor revealed a significant main effect of time $\left(\mathrm{F}_{1,31}=3.85, p=0.05\right)$. Post-hoc comparisons using the Tukey-Kramer test revealed no significant differences between timepoints. There was no main effect of group $\left(\mathrm{F}_{1,31}=1.56, p=0.22\right)$ or interaction effect between group and time $\left(\mathrm{F}_{1,31}=0.10\right.$, $p=0.75)$. Planned contrasts revealed there was no significant difference between the groups at retention $1\left(\mathrm{~F}_{1,31}=3.19, p=0.08\right)$. However, the groups were significantly different at retention $2\left(\mathrm{~F}_{1,31}=5.01, p=0.03\right)$ and when the retention timepoints were pooled together $\left(\mathrm{F}_{1,31}=8.10, p\right.$ $<0.01)$. To represent the size of this difference a percent change from the last 5 trials of posttraining 2 was calculated as the difference between the two groups' normalized retention scores at each time point divided by the average of all the last 5 trials of post-training 2 . At retention 1 this was equivalent to a $10.47 \%$ change from the last 5 trials of post-training 2 , at retention 2 this was a $13.13 \%$ change, and when the retention timepoints were pooled together this was a $11.80 \%$ change.

The transfer test data was also normalized to the last 5 trials of post-training 2 to provide a reference of performance at the end of visit 1 . To analyze the transfer test data a two-tailed $t$ test was run to compare groups at transfer 1 and transfer 2. Results revealed no significant difference between the groups at transfer $1\left(\mathrm{t}_{31}=-0.23, p=0.81\right)$ or at transfer $2\left(\mathrm{t}_{31}=1.36, p=\right.$ $0.18)$. 


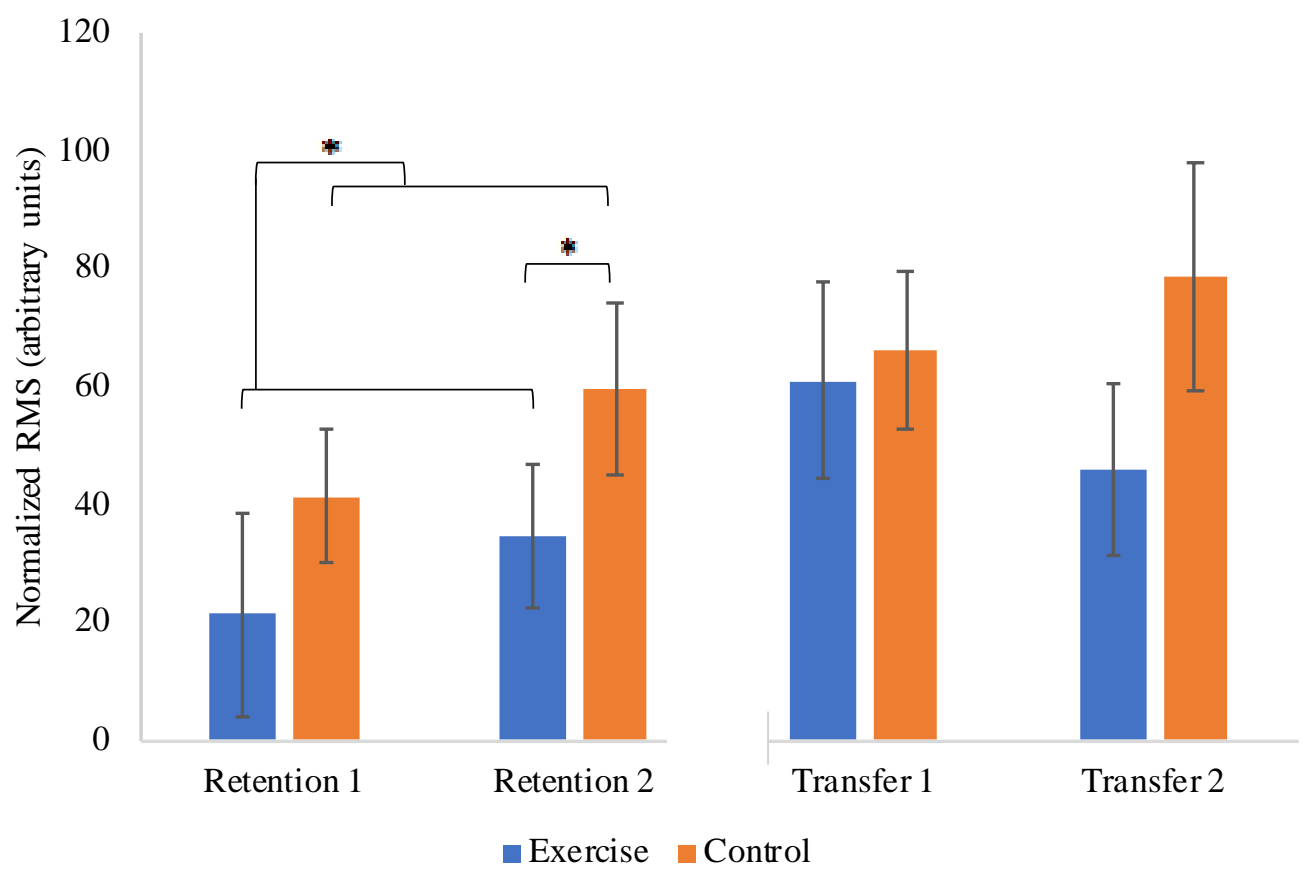

Figure 5. Change in RMS from the last 5 trials of post-training 2 to retention and transfer. Data was normalized to show each group's performance as the difference from the 5 last trials of post-training 2. A lower score is representative of a better performance. Error bars represent standard error of the mean. * indicates significant difference $\mathrm{p}<0.05$.

A mixed-model ANOVA on the RMS acquisition and post-training measures (Figure 6) with time (baseline, early training, late training, post-training 1, post-training 2) as the withinsubjects factor and group $(\mathrm{EXE} / \mathrm{CON})$ as the between-subjects factor revealed a significant main effect of time $\left(\mathrm{F}_{4,124}=98.29, p<0.01\right)$. Post-hoc comparisons using the Tukey-Kramer test revealed that all timepoints were significantly different from one another except for post-training 1 and post-training 2, and late-training and post-training 2 . There was no significant main effect of group $\left(\mathrm{F}_{1,31}=0.05, p=0.81\right)$ and no significant interaction between group and time $\left(\mathrm{F}_{4,124}=\right.$ 1.07, $p=0.37)$. 


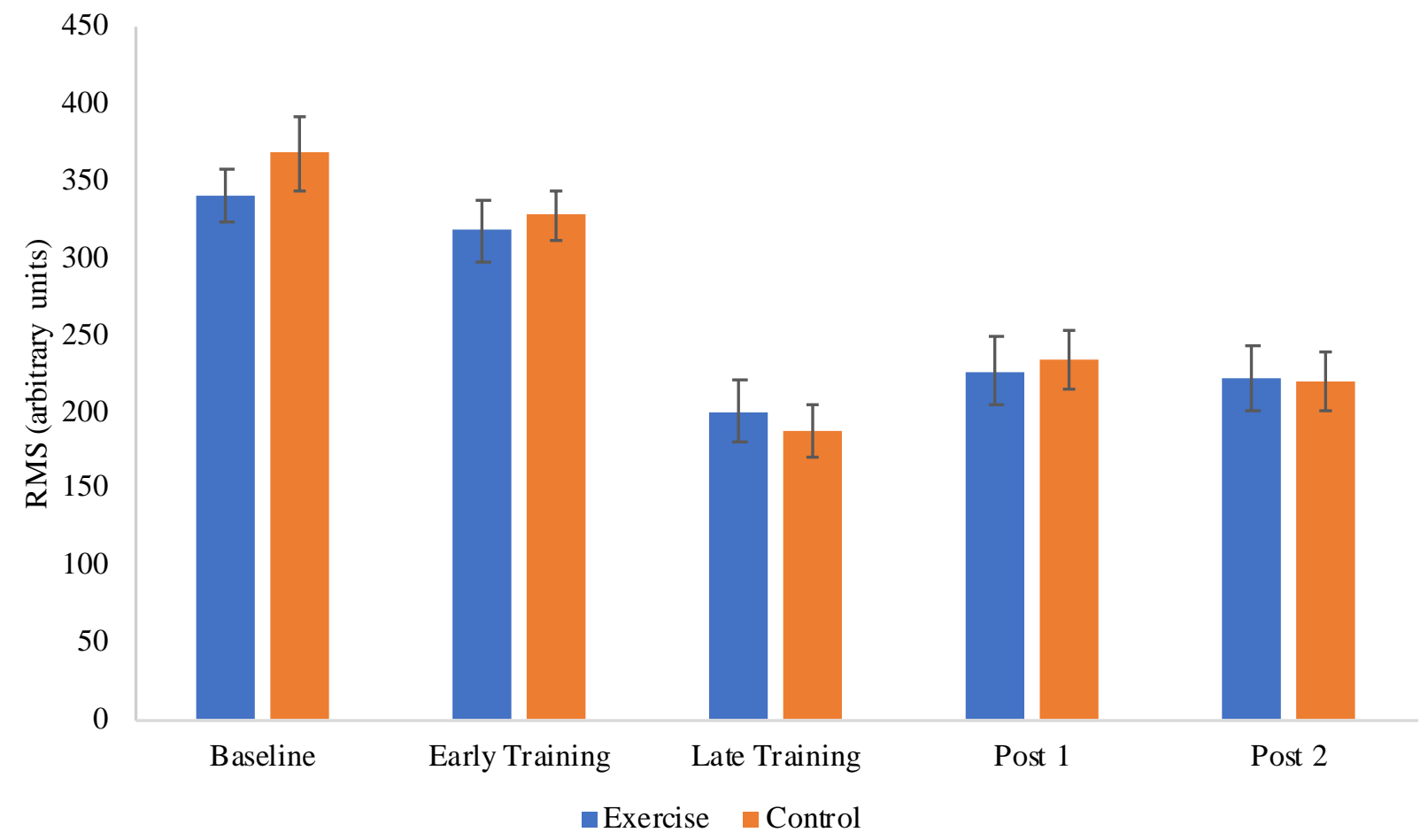

Figure 6. RMS scores for baseline, training, and post-training tests. A lower score is representative of a better performance. Error bars represent standard error of the mean.

A mixed-model ANOVA on the RT retention data (Figure 7) with time (retention 1, retention 2) as the within-subjects factor and group $(\mathrm{EXE} / \mathrm{CON})$ as the between-subjects factor revealed no significant main effects of time $\left(\mathrm{F}_{1,31}=0.46, p=0.50\right)$, group $\left(\mathrm{F}_{1,31}=0.01, p=0.90\right)$, and no significant interaction between group and time $\left(\mathrm{F}_{1,31}=2.93, p=0.09\right)$. Planned contrasts revealed no significant differences between the groups at retention $1\left(\mathrm{~F}_{1,31}=1.12, p=0.29\right)$, retention $2\left(\mathrm{~F}_{1,31}=1.85, p=0.18\right)$, or when the retention timepoints were pooled together $\left(\mathrm{F}_{1,93}=\right.$ $0.05, p=0.83$ ). A two-tailed t test was run on the normalized RT transfer data to compare groups at transfer 1 and transfer 2. Results revealed no significant difference between the groups at transfer $1\left(\mathrm{t}_{31}=-0.60, p=0.55\right)$ or transfer $2\left(\mathrm{t}_{31}=-0.26, p=0.79\right)$. 


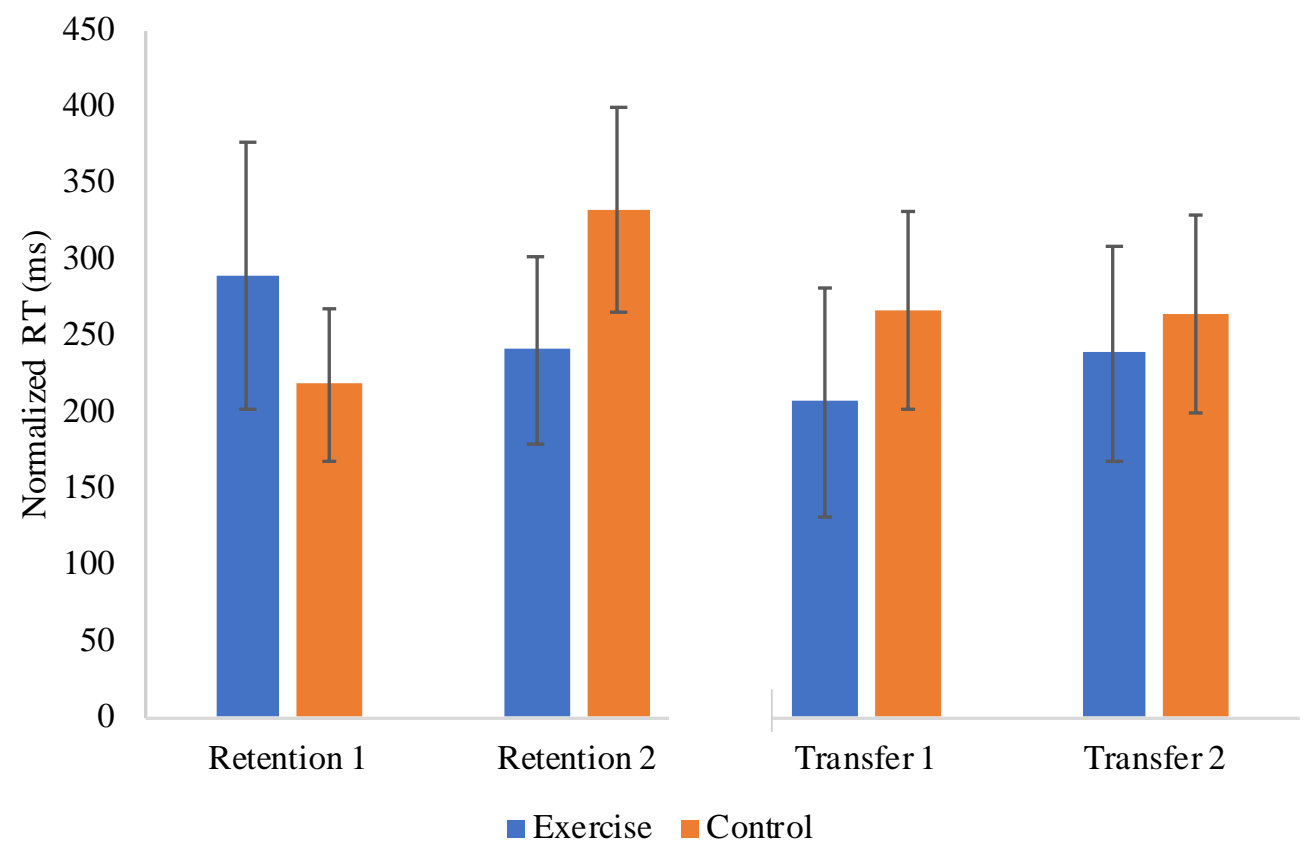

Figure 7. Change in RT from late training to post/retention/transfer. Data was normalized to show each group's performance as the difference from their late training score. A lower score is representative of a better performance. Error bars represent standard error of the mean.

A mixed-model ANOVA on the RT acquisition and post-training measures (Figure 8) with time (baseline, early training, late training, post-training 1, post-training 2) as the withinsubjects factor and group $(\mathrm{EXE} / \mathrm{CON})$ as the between-subjects factor revealed a main effect of time $\left(\mathrm{F}_{4,124}=115.9, p<0.01\right)$. Post-hoc comparisons using the Tukey-Kramer test revealed that baseline and early training were not significantly different from each other, but they were significantly different from every other timepoint. Late training, post-training 1 and post-training 2 were not significantly different from each other. There was no significant main effect of group $\left(\mathrm{F}_{1,31}=0.14, p=0.71\right)$ and no significant interaction between group and time $\left(\mathrm{F}_{4,124}=0.18, p=\right.$ 0.94). 


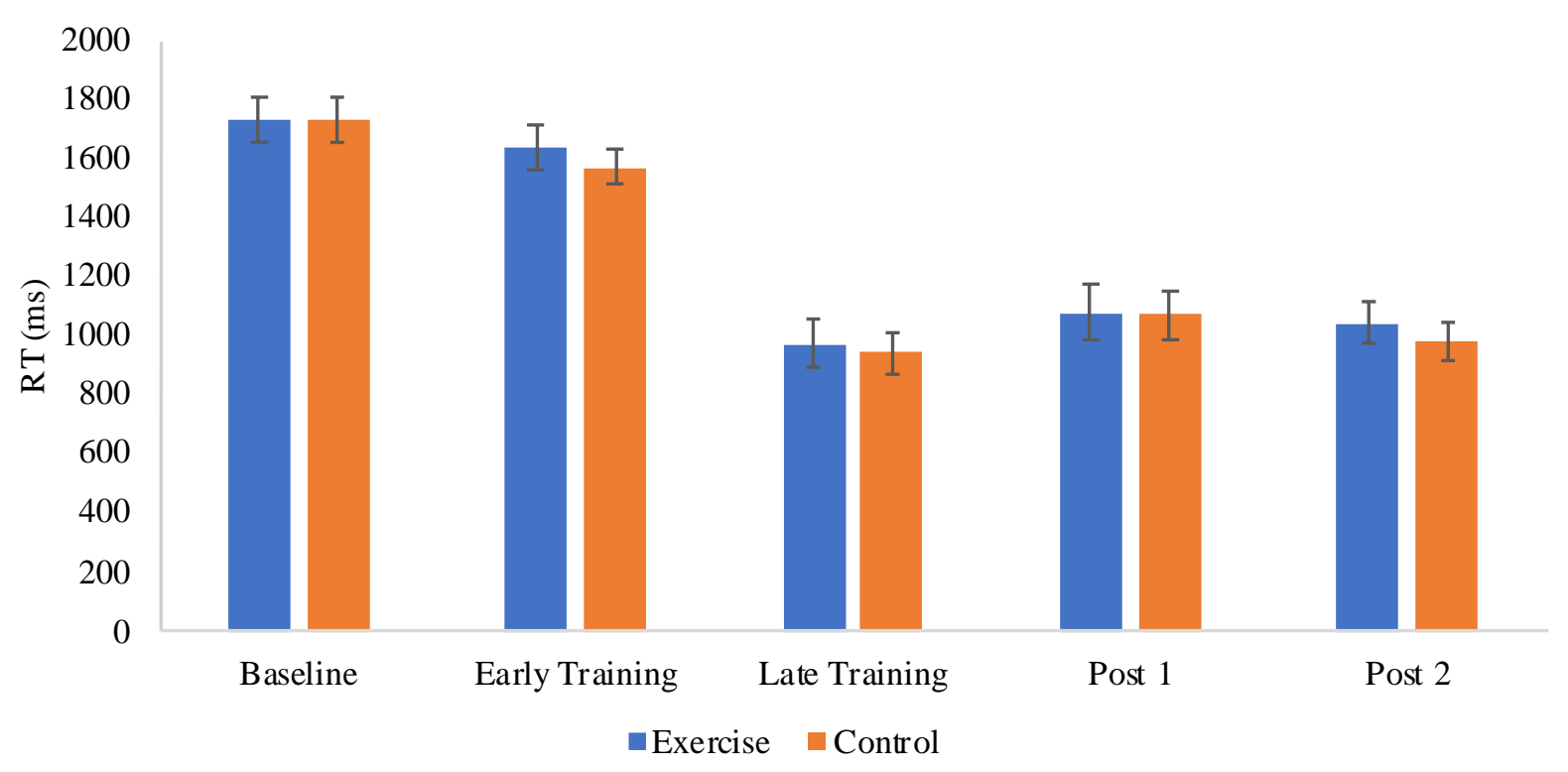

Figure 8. RT scores for baseline, training, and post-training tests. A lower score is representative of a better performance. Error bars represent standard error of the mean.

\subsection{Neurophysiological Data}

A mixed-model ANOVA with the within-subjects factor as time (early training, late training, and post-training 2) and the between-subjects factor as group (EXE/CON) was run on the data from each electrode (FCZ and CZ). Results from the FCZ electrode (Figure 9) revealed a main effect of time $\left(\mathrm{F}_{2,62}=10.33, p<0.01\right)$. Post-hoc comparisons using the Tukey-Kramer test revealed that post-training 2 was significantly different than early training and late training. There were no main effects of group $\left(\mathrm{F}_{1,31}=0.06, p=0.80\right)$ and no interaction between group and time $\left(\mathrm{F}_{2,62}=2.52, p=0.08\right)$. The planned contrast between early and late training was not significant $\left(\mathrm{F}_{1,62}=3.19, p=0.07\right)$. Grand average traces of EXE and CON are displayed in Figure 10a and 10b respectively. Results from the CZ electrode (Figure 11) also showed a main effect of time $\left(\mathrm{F}_{2,62}=3.71, p=0.03\right)$. Post-hoc comparisons using the Tukey-Kramer test revealed that post-training 2 was significantly different than late training. There were no main effects of group $\left(\mathrm{F}_{1,31}=0.23, p=0.63\right)$ and no interaction between group and time $\left(\mathrm{F}_{2,62}=0.92\right.$, 
$p=0.40)$. The planned contrast between early and late training was significant $\left(\mathrm{F}_{1,62}=4.68, p=\right.$

0.03). Grand average traces of EXE and CON are displayed in Figure 12a and 12b respectively.

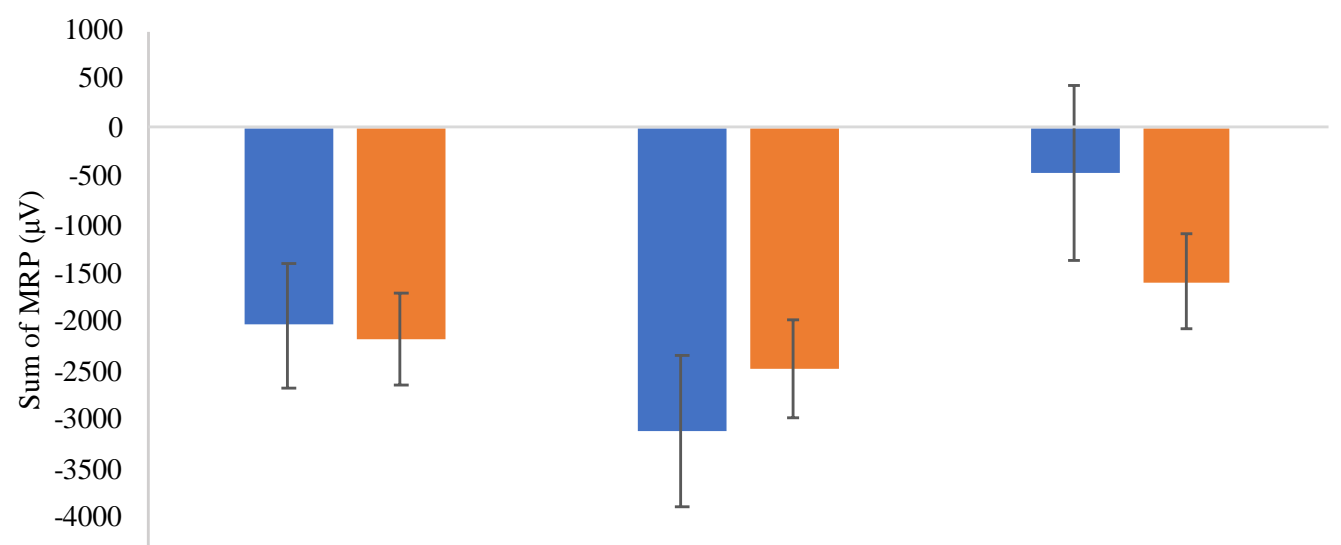

Early Training

Late Training

Post 2

Exercise $\quad$ Control

Figure 9. Sum of all individual data points from MRP trace recorded at FCZ. Error bars represent standard error of the mean.

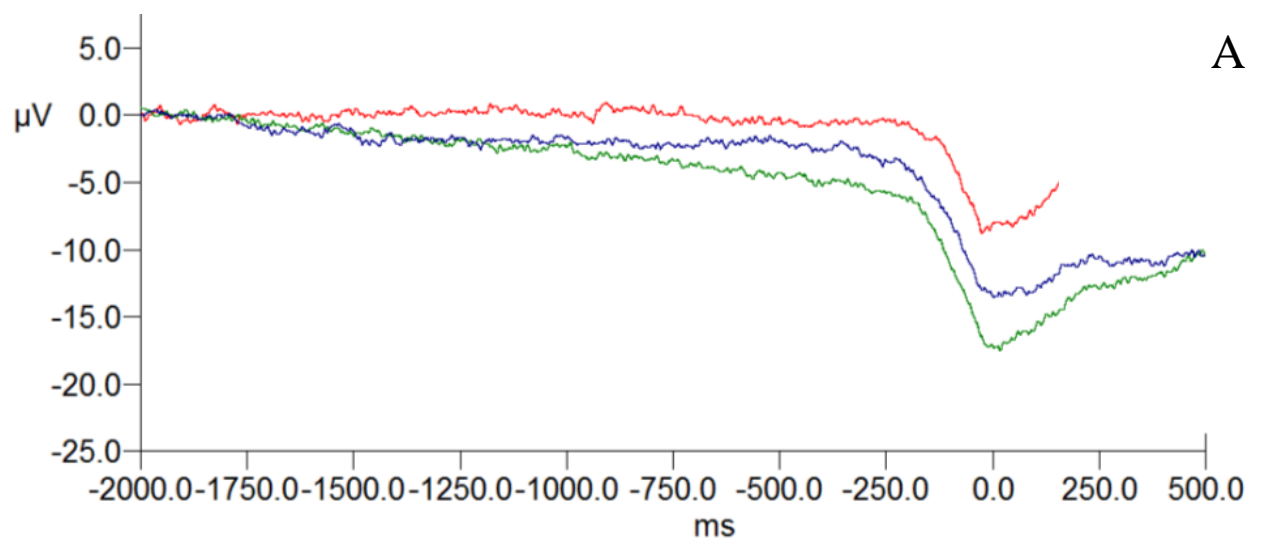

Early Training

Late Training

Post-Training Test 2

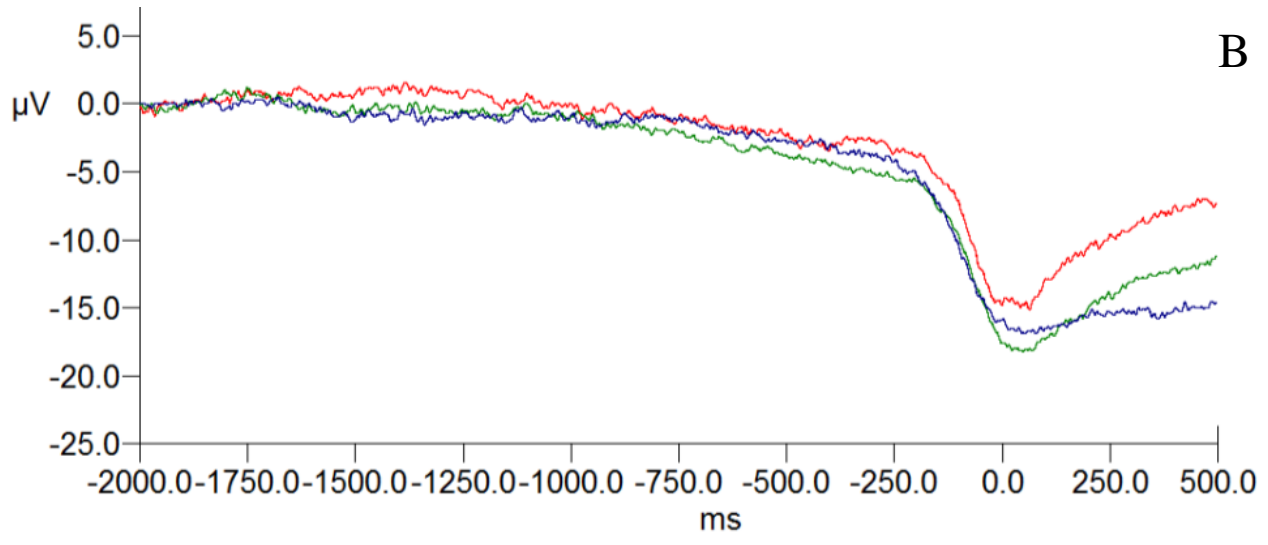

Figure 10. Grand-average MRP trace of EXE (A) and CON (B) recorded from electrode FCZ. Blue represents early training, green represents late training, and red represents post-training test 2. Data is time-locked to cursor movement; 0 represents the start of cursor movement. 


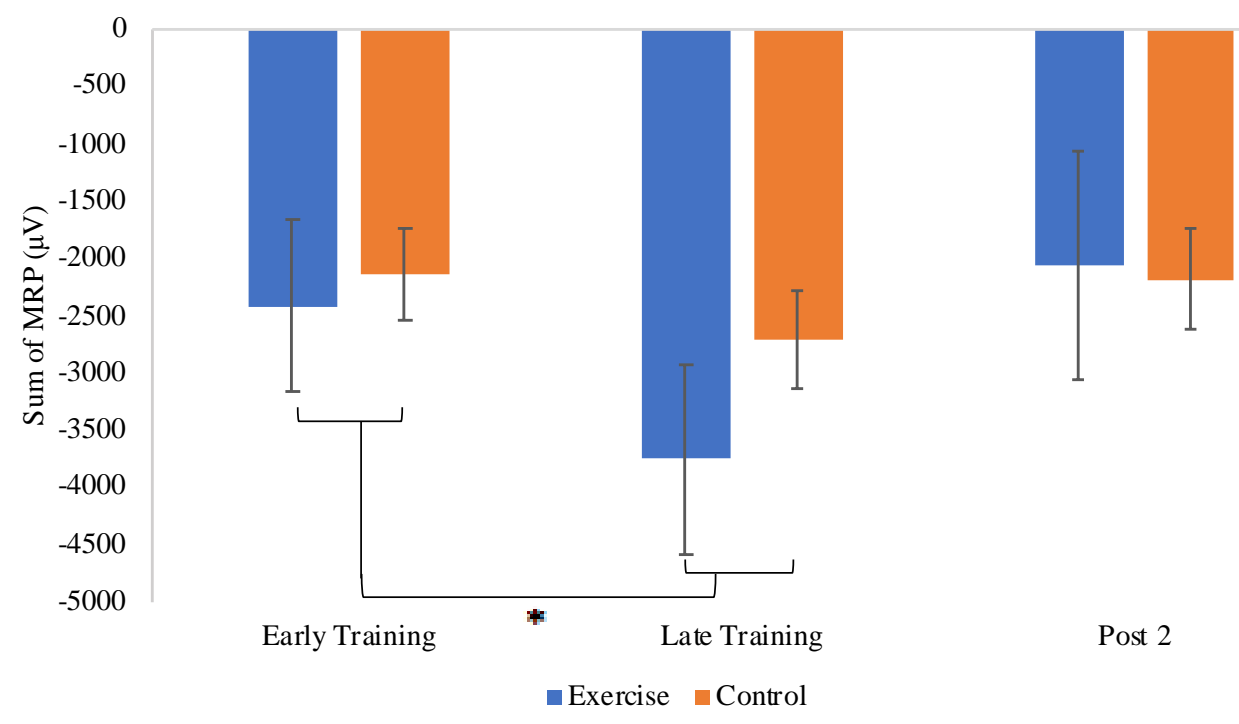

Figure 11. Sum of all individual data points from MRP trace recorded at CZ. Error bars represent standard error of the mean. * indicates significant difference $\mathrm{p}<0.05$.

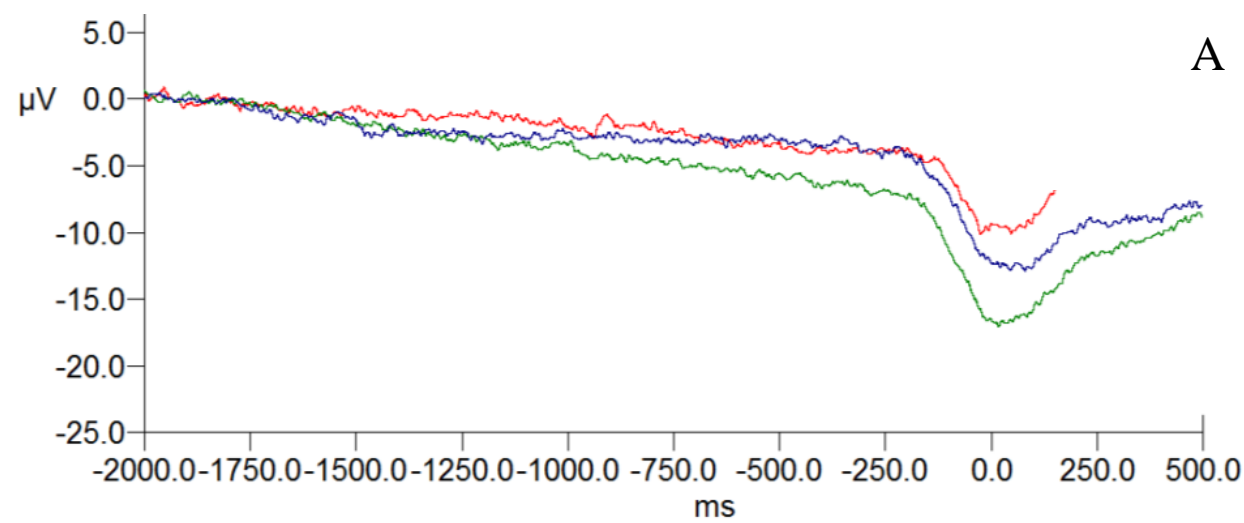

Early Training
Late Training
Post-Training Test 2

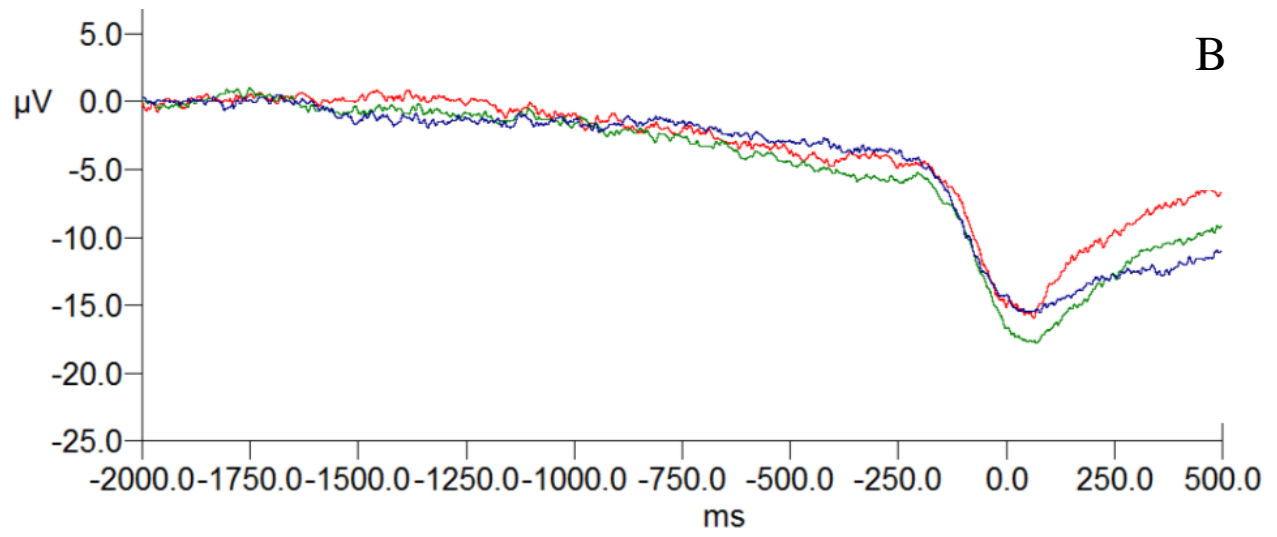

Figure 12. Grand-average MRP trace of EXE (A) and CON (B) recorded from electrode CZ. Blue represents early training, green represents late training, and red represents post-training test 2. Data is time-locked to cursor movement; 0 represents the start of cursor movement. 


\subsection{Correlational Data}

Initially we predicted that in both groups there would be a larger MRP amplitude at late training and post-training 2 than early training. Our results show an increase in the MRP amplitude from early to late training however the MRP amplitude at post-training 2 was not significantly different than early training at the $\mathrm{CZ}$ electrode, and was significantly smaller than the early training at the FCZ electrode. Given these results we examined the relationship between the change in the sum of the MRP amplitude from early to late training and the change in RMS and RT from the last 5 trials of post-training 2 to the retention time points. This allowed us to examine whether changes in the MRP amplitudes during motor training were associated with performance at retention scores. In other words, the degree to which the neural representation of skill acquisition predicted participants' ability to consolidate (or retain) the skill. A Pearson correlation and significance test was run on each comparison. To test for significance a t value was calculated from the coefficient and a two-tailed $t$ test was conducted. There was a positive linear relationship in EXE between the change in MRP amplitude sum recorded from early to late training and the change in RT from the last 5 trials of post-training 2 to retention 1 . This correlation was significant at both FCZ $(r=0.77, p<0.01)$ (Figure 13) and $\mathrm{CZ}(r=0.59, p=$ 0.01). Additionally, when this relationship was examined with both EXE and CON pooled together, there was a significant correlation at FCZ $(r=0.45, p<0.01)$ and at $\mathrm{CZ}$, this correlation approached significance $(r=0.32, p=0.06)$. We also examined the relationship between the change in the sum of the MRP amplitude from early to late training and the change in RMS and RT from baseline to late training. This allowed us to determine whether behavioural improvements during training were associated with changes in the MRP amplitudes during the motor training. We did not find any significant correlations between these variables. 


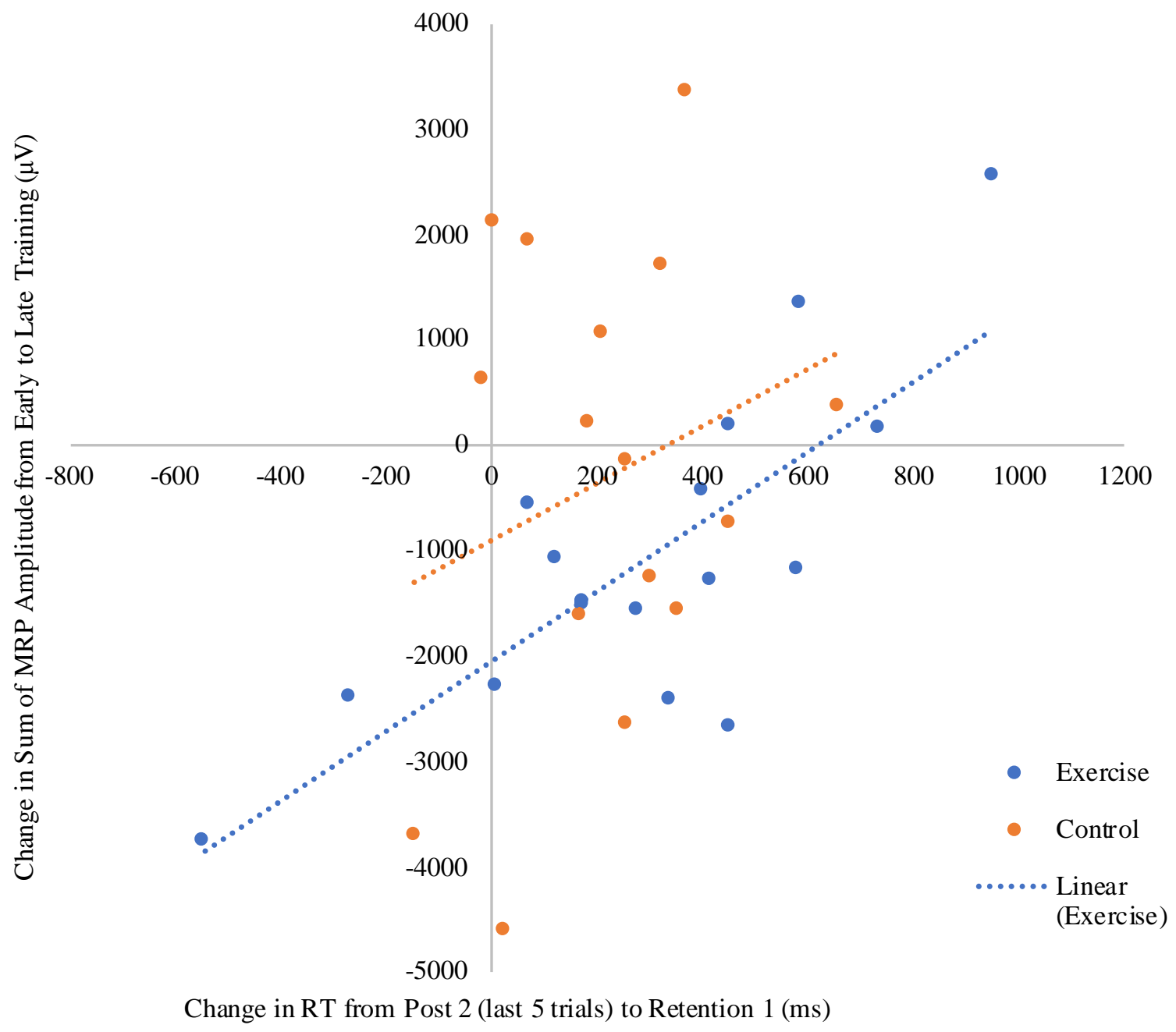

Figure 13. Change in RT at retention vs. change in MRP amplitude. On the $\mathrm{x}$ axis is the change in RT from posttraining 2 (last 5 trials) to retention 1 . A negative score means they performed better than they did at the last 5 trials of post-training 2. On the y axis is the change in the sum of the MRP amplitude at FCZ from early to late training. A negative score means their amplitude increased in the negative direction from early to late training. 


\subsection{Discussion}

The results of our study are generally consistent with the previous studies that have shown that post-motor training high intensity interval training helps to retain performance of a unimanual motor skill, suggesting the timing of this exercise enhances consolidation mechanisms (Roig et al., 2012; Thomas, Flindtgaard, et al., 2016; Thomas, Beck, et al., 2016; Thomas, Johnsen, et al., 2016). Our study adds to the literature in the following ways. First, we show that continuous moderate intensity exercise also provides a benefit to the retention of a motor skill. Second, we show that exercise is able to help retain a more complex, bimanual motor task. Finally, we attempt to correlate neural modulations with behavioural performance at retention tests. Our results provide some evidence that exercise enhances the relationship between increases in excitability during training and performance of the skill at retention.

The main objective of our study was to examine whether a session of continuous moderate intensity aerobic exercise performed after motor training would affect motor memory consolidation as measured by performance at retention measures. Our results support the main hypothesis that EXE would have a significantly lower change in RMS scores compared to CON from the last 5 trials of post-training 2 to retention. However, EXE did not have a significantly lower change in RT score than $\mathrm{CON}$ from the last 5 trials of post-training 2 to retention. In addition, our results support our prediction that both EXE and CON would have significant improvements in RMS and RT at late training and post-training measures and that at these time points there would be no significant difference between the groups. Our second objective was to investigate the influence of motor training and aerobic exercise on cortical activity relating to MRPs. Our main hypothesis that EXE would have a larger amplitude increase of the early MRP component at the post-training measure compared to CON was not supported. We also predicted 
that both groups would have a larger amplitude of the early MRP component at the late training measure compared to the early training measure. This was observed however it was only supported with statistical significance at the $\mathrm{CZ}$ electrode. Finally, we wanted to investigate the relationship between the neural markers of skill acquisition and the consolidation of the skill measured through retention tests. We predicted there would be a correlation between increases in the amplitude of the early MRP component and changes in the RMS and RT score from the last 5 trials of post-training 2 to retention. There was a significant correlation found between the change in MRP amplitude sum from early to late training and the change in RT from the last 5 trials of post-training 2 to retention 1. This was found in EXE, and when EXE and CON were pooled together, but not in CON alone which provides some support for this final hypothesis.

\subsection{Participant Characteristics}

The two groups had similar descriptive characteristics, BMI, MET-minutes per week, and resting HR values, giving some indication that the groups were comparable. Initially we had a high cut-off for the IPAQ scale that excluded individuals in the highly physically active category. However, due to the variability around the IPAQ categorization we choose not to include this. Therefore, the only IPAQ exclusion criteria was accumulating less than 600 METminutes per week which was maintained to exclude individuals who have a very low level of physical activity and may not be able to complete 20 minutes of moderate intensity exercise.

\subsection{Supplementary Data}

The supplementary data collected from the SSS and SMHSQ does not suggest there would be differences between the groups. However, data from PANAS shows a significant difference between the groups at the pre-intervention (before exercise). EXE had a higher mood score, meaning they were in a more positive mood. Additionally, EXE had a post-intervention 
(after exercise) score that was significantly higher than every other score in both groups except for the EXE pre-intervention score. This suggests that EXE had a more positive mood prior to beginning the study, and that this mood was maintained after completing motor training and exercise. There are no known studies that have shown that mood affects motor acquisition or motor memory consolidation. However, mood can contribute to cortical arousal and cognition which may impact how well the task is performed (Mitchell \& Phillips, 2007). Our data show that behaviourally the groups were no different during the motor training session. Therefore, it is unlikely that the differences in mood affected motor acquisition or consolidation.

\subsection{Exercise Data}

Participants in the exercise group were required to complete 20 minutes of continuous moderate intensity exercise on a recumbent bike. Both the average percentage of HRR reached during exercise and the average RPE during exercise reflected the targets set for moderate intensity. All participants maintained RPM within the set range, so we are confident that this variable was controlled for. At post-training 1, HR was still elevated in EXE, but at post-training

2, HR was back to near-resting levels. Our estimation that it would take approximately 30 minutes after exercise for HR to get back to resting was correct as both groups had the same average amount of time from exercise to post-training 2 . The standard deviation was larger in the EXE group which reflects the variability in the time it took for HR to go down from person to person.

\subsection{Behavioural Data}

Performance of the task during acquisition, retention, and transfer was assessed through measures of accuracy (RMS) and speed (RT). We hypothesized that exercise would have the same benefit for both RMS and RT performance because performance of speed and accuracy in 
the task are largely related. As individuals improve at locating the target with the cursor they should be able to quicken their movement to the target. The object of the task was to simultaneously flex both wrists to move the cursor diagonally from the bottom right of the screen to the target in the top left within the $2 \mathrm{~s}$ timeframe. During the first few trials participants may have prioritized speed to be able to reach the cursor within the $2 \mathrm{~s}$. However, once this was achieved participants likely prioritized accuracy. Therefore, the primary measure of performance was RMS scores.

The ANOVA with our RMS retention data showed a main effect of time but the post-hoc did not reveal any significant differences between retention 1 and retention 2 . As well there was no main effect of group or interaction between group and time. Our hypothesis tests revealed that the retention of the skill in CON was significantly worse than EXE at retention 2 and when the retention time points were pooled together. This significant difference between groups was not observed at retention 1 . These results suggest that the benefit of exercise post-motor training is that it helps to consolidate a motor memory so that is resistant to degradation over time. At retention $1 \mathrm{CON}$ does not retain the skill as well as EXE, however there is no significant difference between the groups at this timepoint. It seems likely that the motor memory formed in $\mathrm{CON}$ is still strong 1 day after motor training and therefore our hypothesis test comparing the groups at this timepoint did not reach significance. As a longer period of time goes by, this motor memory may degrade at a faster pace in CON than in EXE. This is likely why at retention 2, one week post-motor training, the retention score of CON is significantly worse than EXE.

The transfer tests were not consistent with the retention test results. The groups are not significantly different from each other at transfer 1 or transfer 2. However, EXE appears to 
improve from transfer 1 to transfer 2, and CON appears to get worse. This suggests that there may be a learning effect in EXE from transfer 1 to transfer 2 that is not present in CON.

The above results suggest that post-motor training moderate intensity exercise assists with retaining the accuracy of the motor skill. While the neural mechanisms of this behavioural benefit cannot be determined from this study, previous studies suggest that exercise increases the availability of LTP-related compounds (Skriver et al., 2014) and assists with the induction of LTP by reducing cortical inhibition and increasing excitation (Singh, Neva, et al., 2014). Studies with similar motor training structures to ours have suggested that BMT can induce early LTPlike plasticity (Neva et al., 2012). Post-motor training exercise may then enhance these BMTinduced plasticity mechanisms by increasing the availability of LTP-related compounds throughout consolidation. Examples of these LTP-related compounds include BDNF, catecholamine neurotransmitters, and lactate. These compounds assist with tagging synapses to undergo the transition from E-LTP to L-LTP. It could be through these mechanisms that exercise enhances motor memory consolidation resulting in a robust motor memory that is resistant to degradation over time.

There were no differences between the groups at baseline, training, or post-training RMS scores (Figure 6). This suggests that both EXE and CON had similar performance levels at these timepoints and that the motor training improved all participants' RMS performance from their baseline scores to early training and to late training timepoints (Figure 6). This confirms that participants were able to improve their accuracy during the acquisition of the skill. As predicted, there were no differences between the two post-training tests, and between late training and posttraining 2. We did not expect there to be a difference from late training to the post-training tests or between the post-training tests as previous research has demonstrated that performance 
differences between groups do not emerge immediately after the intervention (Lundbye-Jensen et al., 2017; Roig et al., 2012). Consolidation has not yet occurred at the time of the post-training tests as post-training 1 occurs 50 minutes after training, and the post-training 2 occurs roughly an hour and 20 minutes after training. Therefore, if post-motor training exercise enhances the consolidation of the motor memory, a difference in performance between the groups at these timepoints is not to be expected.

The results of our analyses on the RT retention data were not consistent with the RMS retention data. There were no main effects of time or group and no interaction. Our hypothesis was not supported as there were no significant differences between the groups at the retention tests. There were also no significant differences between the groups at the transfer tests.

Consistent with the RMS acquisition and post-training data there were no differences between the groups' RT scores at baseline, training, or post-training (Figure 8). Both groups improved their RT from baseline and early training to late training. Their performance at late training then stayed consistent at the post-training tests. These findings confirm that participants improved their speed over the acquisition of the skill, and that there were no immediate performance benefits as predicted.

These results show that by the end of the motor training all participants had improved their performance in both speed and accuracy, however, at the retention tests, speed and accuracy of the skill were not equally retained. When the speed-accuracy data is plotted, there is a positive linear relationship during training and at the retention timepoints. That is, participants who had lower RMS scores also had lower RT scores. Therefore, it does not appear that this finding is a result of a speed-accuracy trade-off. It could be that a few higher RT scores in the data that may have increased the variability in EXE at the retention tests, especially at retention 1. This likely 
had some contribution to the lack of effect observed in speed but the main reason for not seeing a significant difference in speed between the groups at the retention timepoints is likely due to the design of the task. A timeframe of $2 \mathrm{~s}$ was given to complete the task in order to increase the difficulty of the task and to ensure that the planned movement was performed relatively quickly. If they did not reach the target within the $2 \mathrm{~s}$ timeframe their RT was recorded as $2000 \mathrm{~ms}$. Therefore, the range of the RT scores may have been too narrow to see a significant difference between training and retention.

\subsection{Neurophysiological Data}

Our main hypothesis for our second objective was not supported as EXE did not have a larger amplitude increase at the post-training measure compared to CON. In fact, our results showed that the MRP amplitudes decreased for both groups at the post-training measure. At the FCZ electrode the main effect of time revealed that the amplitude at post-training 2 was significantly smaller than early and late training. At CZ the main effect of time showed that posttraining 2 was significantly smaller than late training, and that there was no difference between early training and post-training 2 . We did not observe an effect of exercise on the cued-MRP. This is inconsistent with Thacker et al. (2014) who found an earlier onset of the BP component post-exercise when HR returned to resting. Had we observed an earlier onset of the cued-MRP it would have been reflected in a larger sum of the MRP amplitude. However, differences in the study objectives and tasks likely contributed to the contrasting results. Our study had a motor learning component as our objective was to examine the consolidation of motor memories. Thacker et al. (2014) examined the effects of exercise on motor cortical areas. Considering these objectives, our task was more complex, requiring participants to complete cued bimanual movements to control a cursor on screen, whereas Thacker et al. (2014) had participants 
complete self-paced unimanual wrist extension movements. Cued and self-paced MRPs have slightly different cortical generators and the complexity of our task likely required additional cortical input from other brain areas. Therefore, it may not have been as easily enhanced through exercise. There are many factors that could have prevented us from observing the increased excitability in the motor preparatory areas post-exercise. Changes in the position of the electrode and changes in the impedance of electrodes are possible but unlikely as the cap was kept on during exercise and was not adjusted at any timepoint. Sweat or movement of the gel during exercise may have changed the impedance of the electrodes however impedance was checked in EXE and CON prior to beginning post-training test 2. Although our main hypothesis for our neurophysiological data was not supported, it does not mean that exercise does not enhance consolidation mechanisms. This was the first study to measure MRP modulations in attempt to examine the effects of post-motor training exercise on the consolidation of motor memories. Perhaps measurement of MRP modulations is not the best way to examine this relationship. Instead, analysis of the spectral components may be better suited to assess markers of consolidation such as the work by Dal Maso et al. (2018).

In addition to our main hypothesis we predicted from previous research that there would be an increase in the MRP amplitude from early to late training in both groups (Smith \& Staines, 2010; Smith \& Staines, 2006). This increase in amplitude is thought to be a neural marker for skill acquisition. The hypothesis test contrasting early and late MRP amplitudes across all participants showed that the late training MRP amplitude was significantly greater than the early training MRP amplitude when measured from the CZ electrode. This relationship between early and late training MRP amplitudes was also observed at FCZ, however the magnitude of this was much smaller and therefore did not reach significance. As the early MRP is largely generated 
from activity in premotor and supplementary motor areas, we would expect the maximal MRP amplitude to be captured by FCZ. It is possible that anatomical variability as well as variability in the EEG cap placement may have contributed to slightly higher amplitude recordings at CZ rather than FCZ. Additionally, differences in baseline activity and arousal levels across participants could have resulted in varied initial MRP amplitudes and therefore different changes in MRP amplitudes from early to late training. Despite only being significant at CZ, these results provide evidence that the motor training caused some neural modulations. Our hypothesis that both groups would have a larger amplitude of the early MRP component at the late training compared to the early training was partially supported. An increase in amplitude from early to late training is reflective of increased excitability. As discussed above, this increased excitability is not necessarily a marker of LTP but is likely caused by a reduction in GABA-mediated inhibition and an increase in EPSPs, which in turn increases the likelihood of LTP occurring. Though we cannot conclude that LTP was occurring during motor training, it is possible that motor training started the induction of LTP in both EXE and CON, and this LTP may have been enhanced and prolonged by exercise in EXE.

\subsection{Correlational Data}

Our correlational analysis revealed a significant correlation between the change in the MRP amplitude sum from early to late training and the change in RT from the last 5 trials of post-training 2 to retention 1 . Specifically, the greater the MRP amplitude increase, the better the change in RT score from the last 5 trials of post-training 2 to retention 1 . This correlation was only observed in EXE and when both groups were pooled together. This provides some evidence that there is an underlying relationship between increases in the MRP amplitude during training and the ability to maintain performance of the skill. Since this relationship was only observed in 
EXE and when EXE and CON were pooled together, it suggests that this relationship may be present in both groups, and it is strengthened when exercise helps to consolidate these neural changes to translate them to performance of the skill.

There was no observed relationship between the change in the sum of the MRP amplitude from early to late training and the change in RMS and RT from baseline to late training. Thus, the behavioural improvements during training were not associated with changes in the MRP amplitudes during the training. This contrasts a finding from a previous study that correlated increases in the amplitude of the BP with improvements in the speed of the task during training and when behavioural performance plateaued, the BP amplitude stopped increasing (Taylor, 1978). Task differences likely contributed to our contrasting results. Taylor (1978) required participants to learn a single series button press with 6 buttons and perform it as fast as they could every 20 s. Participants in this task would have prioritized speed and would have improved relatively quickly. Our task was more complex and involved a random variable practice structure to 3 different targets. Random variable practice structure has been shown to improve the retention of the skill but can be of detriment to performance during training. It is also possible that the increase in amplitude from early to late training was not large enough to assess this relationship. Perhaps participants would have had a larger magnitude of behavioural improvement from baseline to late training and a larger amplitude change from early to late training if the same target had been presented each time. A longer training period or a larger sample size may be needed to observe a significant correlation between improvements in our task during training and amplitude increases from early to late training.

These results lead to the question of whether exercise performed prior to and after training would be of benefit for the retention of the motor skill. There is some evidence that 
exercise performed prior to motor training improves the acquisition of the skill (Snow et al., 2016; Statton et al., 2015). Our data provides some evidence that individuals with larger neural modulations as a result of training are better able to retain the speed of the skill. Therefore, if pre-motor training exercise enhanced these neural modulations during training then it could be that exercising before and after motor training would further improve the retention of the skill. Other movement-related neural markers that could be used to examine this relationship include beta-band ERD. Measuring beta-band ERD may provide additional insight into changes in movement preparation over motor training as well as post-exercise. Previous studies have shown that beta-band ERD is altered by motor training structure and movement parameters (Park, Kim, \& Chung, 2013; Tzagarakis, Ince, Leuthold, \& Pellizzer, 2010), and post-motor training exercise (Dal Maso et al., 2018). 


\subsection{Limitations}

Only collecting EEG during the first visit limited our ability to correlate neural activity at retention with behavioural measures at these timepoints. However, comparing EEG measurements across different days would be challenging as there are many variables that contribute to the amplitude of the recorded MRP. For example, baseline neural activity, impedance, and position of the electrodes. Day-to-day individuals may have different arousal levels thus we would not have been able to say with confidence that the measurements were comparable. If we were able to control for all of these variables, we would have expected to see larger MRP amplitudes relative to early training for participants in EXE who were able to retain their performance. Since CON did not retain their performance as well as EXE, we would have expected CON to have a smaller MRP amplitude than EXE. Due to this limitation of EEG we only attempted to correlate retention scores with neural activity during the initial consolidation period after motor training and exercise.

We did not account for fitness levels besides excluding individuals with low physical activity levels. While physical activity levels and fitness are correlated, they are not synonymous. The IPAQ was used to get an idea of participant's physical activity levels based on their METs per week. However, as with any self-report measure, the IPAQ is subjective and is not as reliable as an objective measurement of fitness. 


\subsection{Conclusions and Future Directions}

Our main finding from this study is that moderate intensity exercise performed postmotor training assists with the retention of the motor skill. This is an important finding for sports and rehabilitation applications as it suggests that exercise could be used as an adjunct to motor practice. Specific recommendations for coaches and clinicians include structuring practice sessions to first involve practicing a specific technical skill and completing aerobic exercise after this practice. Our results suggest that this may promote consolidation mechanisms to prevent the motor memory from degrading over practice sessions that occur days apart. One recent study had stroke patients complete high intensity interval training post-motor training and found a benefit to motor skill retention (Nepveu et al., 2017). While some neurorehabilitation patients may be able to participate in high intensity exercise, not all may be deemed safe to do so. Previous research has suggested that low intensity exercise provides a benefit, but high intensity exercise provides a greater benefit (Thomas, Johnsen, et al., 2016). If there is this proposed dose-response relationship between exercise intensity level and retention benefit, moderate intensity exercise should provide benefits that are greater than low intensity but less than high intensity. Neurorehabilitation patients who are not able to safely participate in high intensity exercise may start with low intensity exercise, and eventually use moderate intensity exercise to progress to be able to participate in high intensity exercise to get the maximal benefit.

In the future, examining the influence of fitness levels on this relationship between postmotor training exercise on the consolidation of motor memories would be beneficial. To do this a graded exercise test could be used to determine fitness levels. By testing individuals of low, moderate, and high fitness levels we could investigate the interaction between acute and chronic 
exercise to see if post-training exercise equally enhances consolidation for individuals of all fitness levels.

Previous research provides evidence that high intensity interval training can help to retain motor skill performance, likely by enhancing motor memory consolidation. Our results demonstrate that moderate continuous exercise also shows this benefit. Currently, the results do not allow researchers to conclude that one intensity provides more benefit than the other. High intensities seem to be more beneficial than low intensities, however, it is not known if the intensity employed in this study is equivalent or worse at retaining motor skill performance than high intensity interval training. To further investigate the relationship between exercise intensity and motor memory consolidation, different intensities should be tested and directly compared.

Finally, future research should look to include individuals in special populations including older adults and neurorehabilitation patients. Currently there is a limited amount of research examining the relationship between exercise and motor learning in older adults, and most of this research examines the effect of chronic exercise (Hübner \& Voelcker-Rehage, 2017). Additionally, in stroke patients, bimanual movements can increase activity between hemispheres (Staines et al., 2001). Considering our results, it is possible that exercising after BMT may enhance this effect. This is a future avenue that should be explored. 


\subsection{References}

Abraham, W. C., \& Bear, M. F. (1996). Metaplasticity: The plasticity of synaptic plasticity. Trends in Neurosciences. https://doi.org/10.1016/S0166-2236(96)80018-X

Aizawa, H., Inase, M., Mushiake, H., Shima, K., \& Tanji, J. (1991). Reorganization of activity in the supplementary motor area associated with motor learning and functional recovery. Experimental Brain Research. https://doi.org/10.1007/BF00230980

Andres, F. G., Mima, T., Schulman, A. E., Dichgans, J., Hallett, M., \& Gerloff, C. (1999). Functional coupling of human cortical sensorimotor areas during bimanual skill acquisition. Brain. https://doi.org/10.1093/brain/122.5.855

Berchtold, N. C., Chinn, G., Chou, M., Kesslak, J. P., \& Cotman, C. W. (2005). Exercise primes a molecular memory for brain-derived neurotrophic factor protein induction in the rat hippocampus. Neuroscience. https://doi.org/10.1016/j.neuroscience.2005.03.026

Berry, R. B., \& Wagner, M. H. (2014). Sleep Medicine Pearls. Sleep Medicine Pearls. https://doi.org/10.1016/C2012-0-03542-9

Borg, G. (1970). Perceived exertion as an indicator of somatic stress. Scandinavian Journal of Rehabilitation Medicine, 2(2), 92-98. https://doi.org/S/N

Borota, D., Murray, E., Keceli, G., Chang, A., Watabe, J. M., Ly, M., .. Yassa, M. A. (2014). Post-study caffeine administration enhances memory consolidation in humans. Nature Neuroscience. https://doi.org/10.1038/nn.3623

Brashers-Krug, T., Shadmehr, R., \& Bizzi, E. (1996). Consolidation in human motor memory. Nature, 382(6588), 252-255. https://doi.org/10.1038/382252a0

Brunia, C. H., \& van Boxtel, G. J. (2001). Wait and see. International Journal of Psychophysiology, 43(1), 59-75. https://doi.org/10.1016/S0167-8760(01)00179-9 
Brünig, I., Penschuck, S., Berninger, B., Benson, J., \& Fritschy, J. M. (2001). BDNF reduces miniature inhibitory postsynaptic currents by rapid downregulation of GABAA receptor surface expression. European Journal of Neuroscience. https://doi.org/10.1046/j.0953816X.2001.01506.x

Bütefisch, C. M., Davis, B. C., Wise, S. P., Sawaki, L., Kopylev, L., Classen, J., \& Cohen, L. G. (2000). Mechanisms of use-dependent plasticity in the human motor cortex. Proceedings of the National Academy of Sciences of the United States of America, 97(7), 3661-3665. https://doi.org/10.1073/pnas.050350297

Butefisch, C. M., Khurana, V., Kopylev, L., \& Cohen, L. G. (2004). Enhancing Encoding of a Motor Memory in the Primary Motor Cortex By Cortical Stimulation. Journal of Neurophysiology. https://doi.org/10.1152/jn.01038.2003

Cantarero, G., Tang, B., O’Malley, R., Salas, R., \& Celnik, P. (2013). Motor Learning Interference Is Proportional to Occlusion of LTP-Like Plasticity. Journal of Neuroscience. https://doi.org/10.1523/JNEUROSCI.4706-12.2013

Cirillo, J., Lavender, A. P., Ridding, M. C., \& Semmler, J. G. (2009). Motor cortex plasticity induced by paired associative stimulation is enhanced in physically active individuals. Journal of Physiology, 587(24), 5831-5842. https://doi.org/10.1113/jphysiol.2009.181834

Cotman, C. W., Berchtold, N. C., \& Christie, L. A. (2007). Exercise builds brain health: key roles of growth factor cascades and inflammation. Trends in Neurosciences. https://doi.org/10.1016/j.tins.2007.06.011

Cunnington, R., Iansek, R., Bradshaw, J., \& Phillips, J. (1996). Movement-related potentials associated with movement preparation and motor imagery. Experimental Brain Research, 111(3). https://doi.org/10.1007/BF00228732 
Dal Maso, F., Desormeau, B., Boudrias, M. H., \& Roig, M. (2018). Acute cardiovascular exercise promotes functional changes in cortico-motor networks during the early stages of motor memory consolidation. NeuroImage, 174, 380-392.

https://doi.org/10.1016/j.neuroimage.2018.03.029

Dayan, E., \& Cohen, L. G. (2011). Neuroplasticity subserving motor skill learning. Neuron. https://doi.org/10.1016/j.neuron.2011.10.008

Del Percio, C., Rossini, P. M., Marzano, N., Iacoboni, M., Infarinato, F., Aschieri, P., ... Eusebi, F. (2008). Is there a "neural efficiency" in athletes? A high-resolution EEG study. NeuroImage. https://doi.org/10.1016/j.neuroimage.2008.05.061

Doyon, J., Song, A. W., Karni, A., Lalonde, F., Adams, M. M., \& Ungerleider, L. G. (2002). Experience-dependent changes in cerebellar contributions to motor sequence learning. Proceedings of the National Academy of Sciences. https://doi.org/10.1073/pnas.022615199

Doyon, J., \& Ungerleider, L. G. (2002). Functional anatomy of motor skill learning. In L. R. Squire \& D. L. Schacter (Eds.), Neuropsychology of memory (3rd ed., pp. 225-238). The Guilford Press.

Doyon, Julien, \& Benali, H. (2005). Reorganization and plasticity in the adult brain during learning of motor skills. Current Opinion in Neurobiology. https://doi.org/10.1016/j.conb.2005.03.004

Doyon, Julien, Penhune, V., \& Ungerleider, L. G. (2003). Distinct contribution of the corticostriatal and cortico-cerebellar systems to motor skill learning. Neuropsychologia. https://doi.org/10.1016/S0028-3932(02)00158-6

Dudai, Y. (2012). The Restless Engram: Consolidations Never End. Annual Review of Neuroscience. https://doi.org/10.1146/annurev-neuro-062111-150500 
Eichenbaum, H. (2016). Still searching for the engram. Learning and Behavior. https://doi.org/10.3758/s13420-016-0218-1

Erickson, K. I., Voss, M. W., Prakash, R. S., Basak, C., Szabo, A., Chaddock, L., ... Kramer, A. F. (2011). Exercise training increases size of hippocampus and improves memory. Proceedings of the National Academy of Sciences, 108(7), 3017-3022. https://doi.org/10.1073/pnas.1015950108

Etnier, J. L., Romero, D. H., \& Traustadottir, T. (2001). Acquisition and Retention of Motor Skills as a Function of Age and Aerobic Fitness. Journal of Aging and Physical Activity.

Farmer, J., Zhao, X., Van Praag, H., Wodtke, K., Gage, F. H., \& Christie, B. R. (2004). Effects of voluntary exercise on synaptic plasticity and gene expression in the dentate gyrus of adult male sprague-dawley rats in vivo. Neuroscience. https://doi.org/10.1016/j.neuroscience.2003.09.029

Floyer-Lea, A., Wylezinska, M., Kincses, T., \& Matthews, P. M. (2006). Rapid Modulation of GABA Concentration in Human Sensorimotor Cortex During Motor Learning. Journal of Neurophysiology. https://doi.org/10.1152/jn.00346.2005

Frey, U., \& Morris, R. G. M. (1998). Synaptic tagging: Implications for late maintenance of hippocampal long- term potentiation. Trends in Neurosciences. https://doi.org/10.1016/S0166-2236(97)01189-2

Galea, J. M., Vazquez, A., Pasricha, N., Orban De Xivry, J. J., \& Celnik, P. (2011). Dissociating the roles of the cerebellum and motor cortex during adaptive learning: The motor cortex retains what the cerebellum learns. Cerebral Cortex, 21(8), 1761-1770. https://doi.org/10.1093/cercor/bhq246

Hermens, H. J., Freriks, B., Disselhorst-Klug, C., \& Rau, G. (2000). Development of 
recommendations for SEMG sensors and sensor placement procedures. Journal of Electromyography and Kinesiology, 10(5), 361-374. https://doi.org/10.1016/S1050$6411(00) 00027-4$

Hillman, C. H., Erickson, K. I., \& Kramer, A. F. (2008). Be smart, exercise your heart: exercise effects on brain and cognition. Nat Rev Neurosci., 9(1), 58-65. https://doi.org/10.1038/nrn2298

Huang, T., Larsen, K. T., Ried-Larsen, M., Møller, N. C., \& Andersen, L. B. (2014). The effects of physical activity and exercise on brain-derived neurotrophic factor in healthy humans: A review. Scandinavian Journal of Medicine \& Science in Sports. https://doi.org/10.1111/sms.12069

Huang, Y.-Z., Chen, R. S., Rothwell, J. C., \& Wen, H. Y. (2007). The after-effect of human theta burst stimulation is NMDA receptor dependent. Clinical Neurophysiology, 118(5), 10281032. https://doi.org/DOI 10.1016/j.clinph.2007.01.021

Hübner, L., \& Voelcker-Rehage, C. (2017). Does physical activity benefit motor performance and learning of upper extremity tasks in older adults? - A systematic review. European Review of Aging and Physical Activity. https://doi.org/10.1186/s11556-017-0181-7

Ito, M., \& Kano, M. (1982). Long-lasting depression of parallel fiber-Purkinje cell transmission induced by conjunctive stimulation of parallel fibers and climbing fibers in the cerebellar cortex. Neuroscience Letters, 33(3), 253-258. https://doi.org/10.1016/0304-3940(82)903809

Izumi, Y., Katsuki, H., \& Zorumski, C. F. (1997). Monocarboxylates (pyruvate and lactate) as alternative energy substrates for the induction of long-term potentiation in rat hippocampal slices. Neuroscience Letters. https://doi.org/10.1016/S0304-3940(97)00567-3 
Jacobs, K., \& Donoghue, J. (1991). Reshaping the cortical motor map by unmasking latent intracortical connections. Science. https://doi.org/10.1126/science.2000496

Jäncke, L., Shah, N. J., \& Peters, M. (2000). Cortical activations in primary and secondary motor areas for complex bimanual movements in professional pianists. Cognitive Brain Research. https://doi.org/10.1016/S0926-6410(00)00028-8

Jueptner, M., Stephan, K. M., Frith, C. D., Brooks, D. J., Frackowiak, R. S. J., \& Passingham, R. E. (1997). Anatomy of Motor Learning. I. Frontal Cortex and Attention to Action. Journal of Neurophysiology. https://doi.org/10.1152/jn.1997.77.3.1313

Kamijo, K., O’Leary, K. C., Pontifex, M. B., Themanson, J. R., \& Hillman, C. H. (2010). The relation of aerobic fitness to neuroelectric indices of cognitive and motor task preparation. Psychophysiology, 47(5), 814-821. https://doi.org/10.1111/j.1469-8986.2010.00992.x

Kantak, S. S., Sullivan, K. J., Fisher, B. E., Knowlton, B. J., \& Winstein, C. J. (2010). Neural substrates of motor memory consolidation depend on practice structure. Nature Neuroscience. https://doi.org/10.1038/nn.2596

Kantak, S. S., Sullivan, K. J., Fisher, B. E., Knowlton, B. J., \& Winstein, C. J. (2011). Transfer of motor learning engages specific neural substrates during motor memory consolidation dependent on the practice structure. Journal of Motor Behavior. https://doi.org/10.1080/00222895.2011.632657

Karni, A., Meyer, G., Jezzard, P., Adams, M. M., Turner, R., \& Ungerleider, L. G. (1995). Functional MRI evidence for adult motor cortex plasticity during motor skill learning. Nature. https://doi.org/10.1038/377155a0

Karni, A., Meyer, G., Rey-Hipolito, C., Jezzard, P., Adams, M. M., Turner, R., \& Ungerleider, L. G. (1998). The acquisition of skilled motor performance: Fast and slow experience-driven 
changes in primary motor cortex. Proceedings of the National Academy of Sciences, 95(3), 861-868. https://doi.org/10.1073/pnas.95.3.861

Kim, B. W., Choi, M., Kim, Y.-S., Park, H., Lee, H.-R., Yun, C.-O., ... Son, H. (2008). Vascular endothelial growth factor (VEGF) signaling regulates hippocampal neurons by elevation of intracellular calcium and activation of calcium/calmodulin protein kinase II and mammalian target of rapamycin. Cellular Signalling, 20(4), 714-725.

https://doi.org/https://doi.org/10.1016/j.cellsig.2007.12.009

King, B. R., Hoedlmoser, K., Hirschauer, F., Dolfen, N., \& Albouy, G. (2017). Sleeping on the motor engram: The multifaceted nature of sleep-related motor memory consolidation. Neuroscience and Biobehavioral Reviews. https://doi.org/10.1016/j.neubiorev.2017.04.026

Kleim, J. A., Hogg, T. M., VandenBerg, P. M., Cooper, N. R., Bruneau, R., \& Remple, M. (2004). Cortical Synaptogenesis and Motor Map Reorganization Occur during Late, But Not Early, Phase of Motor Skill Learning. Journal of Neuroscience. https://doi.org/10.1523/JNEUROSCI.3440-03.2004

Klein, A. B., Williamson, R., Santini, M. A., Clemmensen, C., Ettrup, A., Rios, M., ... Aznar, S. (2011). Blood BDNF concentrations reflect brain-tissue BDNF levels across species. International Journal of Neuropsychopharmacology. https://doi.org/10.1017/S1461145710000738

Kornhuber, H. H., \& Deecke, L. (1965). Hirnpotentialänderungen bei Willkürbewegungen und passiven Bewegungen des Menschen: Bereitschaftspotential und reafferente Potentiale. Pflügers Archiv Für Die Gesamte Physiologie Des Menschen Und Der Tiere, 284(1), 1-17. https://doi.org/10.1007/BF00412364

Korol, D. L., \& Gold, P. E. (2008). Epinephrine converts long-term potentiation from transient to 
durable form in awake rats. Hippocampus. https://doi.org/10.1002/hipo.20372

Kotecha, S. A., Oak, J. N., Jackson, M. F., Perez, Y., Orser, B. A., Van Tol, H. H. M., \& MacDonald, J. F. (2002). A D2 class dopamine receptor transactivates a receptor tyrosine kinase to inhibit NMDA receptor transmission. Neuron, 35(6), 1111-1122. https://doi.org/10.1016/S0896-6273(02)00859-0

Krakauer, J. W. (2006). Motor learning: Its relevance to stroke recovery and neurorehabilitation. Current Opinion in Neurology. https://doi.org/10.1097/01.wco.0000200544.29915.cc

Kramer, A. F., \& Erickson, K. I. (2007). Capitalizing on cortical plasticity: influence of physical activity on cognition and brain function. Trends in Cognitive Sciences. https://doi.org/10.1016/j.tics.2007.06.009

Lang, W., Beisteiner, R., Lindinger, G., \& Deecke, L. (1992). Changes of cortical activity when executing learned motor sequences. Experimental Brain Research, 89(2), 435-440. https://doi.org/10.1007/BF00228259

Léger, L., \& Thivierge, M. (1988). Heart Rate Monitors: Validity, Stability, and Functionality. The Physician and Sportsmedicine, 16(5), 143-151. https://doi.org/10.1080/00913847.1988.11709511

Logothetis, N. K. (2008). What we can do and what we cannot do with fMRI. Nature. https://doi.org/10.1038/nature06976

Lømo, T. (1966). Frequency potentiation of excitatory synaptic activity in the dentate area of the hippocampal formation. Acta Physiol. Scand, 227, 128. https://doi.org/citeulike-articleid:3133405

Lu, Y., Christian, K., \& Lu, B. (2008). BDNF: A key regulator for protein synthesis-dependent LTP and long-term memory? Neurobiology of Learning and Memory. 
https://doi.org/10.1016/j.nlm.2007.08.018

Luck, S. J. (2005). An Introduction to the Event-Related Potential Technique. Monographs of the Society for Research in Child Development. https://doi.org/10.1118/1.4736938

Lulic, T., El-Sayes, J., Fassett, H. J., \& Nelson, A. J. (2017). Physical activity levels determine exerciseinduced changes in brain excitability. PLoS ONE, 12(3). https://doi.org/10.1371/journal.pone.0173672

Lundbye-Jensen, J., Skriver, K., Nielsen, J. B., \& Roig, M. (2017). Acute Exercise Improves Motor Memory Consolidation in Preadolescent Children. Frontiers in Human Neuroscience, 11. https://doi.org/10.3389/fnhum.2017.00182

Maity, S., Rah, S., Sonenberg, N., Gkogkas, C. G., \& Nguyen, P. V. (2015). Norepinephrine triggers metaplasticity of LTP by increasing translation of specific mRNAs. Learning and Memory. https://doi.org/10.1101//m.039222.115

Mang, C. S. S., Snow, N. J. J., Wadden, K. P. P., Campbell, K. L. L., \& Boyd, L. A. A. (2016). High-Intensity Aerobic Exercise Enhances Motor Memory Retrieval. Medicine and Science in Sports and Exercise, (28), 2477-2486. https://doi.org/10.1249/MSS.0000000000001040

Mang, C. S., Snow, N. J., Campbell, K. L., Ross, C. J. D., \& Boyd, L. A. (2014). A single bout of high-intensity aerobic exercise facilitates response to paired associative stimulation and promotes sequence-specific implicit motor learning. Journal of Applied Physiology, 117(11), 1325-1336. https://doi.org/10.1152/japplphysiol.00498.2014

Mang, Cameron S., Brown, K. E., Neva, J. L., Snow, N. J., Campbell, K. L., \& Boyd, L. A. (2016). Promoting Motor Cortical Plasticity with Acute Aerobic Exercise: A Role for Cerebellar Circuits. Neural Plasticity. https://doi.org/10.1155/2016/6797928

McDonnell, M. N., Buckley, J. D., Opie, G. M., Ridding, M. C., \& Semmler, J. G. (2013). A 
single bout of aerobic exercise promotes motor cortical neuroplasticity. Journal of Applied Physiology, 114(9), 1174-1182. https://doi.org/10.1152/japplphysiol.01378.2012

Meehan, S. K., Zabukovec, J. R., Dao, E., Cheung, K. L., Linsdell, M. a, \& Boyd, L. a. (2013). One hertz repetitive transcranial magnetic stimulation over dorsal premotor cortex enhances offline motor memory consolidation for sequence-specific implicit learning. The European Journal of Neuroscience. https://doi.org/10.1111/ejn.12291

Mitchell, R. L. C., \& Phillips, L. H. (2007). The psychological, neurochemical and functional neuroanatomical mediators of the effects of positive and negative mood on executive functions. Neuropsychologia. https://doi.org/10.1016/j.neuropsychologia.2006.06.030

Monte-Silva, K., Liebetanz, D., Grundey, J., Paulus, W., \& Nitsche, M. a. (2010). Dosagedependent non-linear effect of L-dopa on human motor cortex plasticity. The Journal of Physiology, 588, 3415-3424. https://doi.org/10.1113/jphysiol.2010.190181

Muellbacher, W., Ziemann, U., Wissel, J., Dang, N., Kofler, M., Facchini, S., ... Hallett, M. (2002). Early consolidation in human primary motor cortex. Nature, 415(6872), 640-644. https://doi.org/10.1038/nature712

Müssgens, D. M., \& Ullén, F. (2015). Transfer in Motor Sequence Learning: Effects of Practice Schedule and Sequence Context. Frontiers in Human Neuroscience. https://doi.org/10.3389/fnhum.2015.00642

Nachev, P., Kennard, C., \& Husain, M. (2008). Functional role of the supplementary and presupplementary motor areas. Nature Reviews Neuroscience. https://doi.org/10.1038/nrn2478

Nepveu, J.-F., Thiel, A., Tang, A., Fung, J., Lundbye-Jensen, J., Boyd, L. A., \& Roig, M. (2017). A Single Bout of High-Intensity Interval Training Improves Motor Skill Retention in Individuals With Stroke. Neurorehabilitation and Neural Repair. 
https://doi.org/10.1177/1545968317718269

Neuper, C., \& Pfurtscheller, G. (2001). Event-related dynamics of cortical rhythms: Frequencyspecific features and functional correlates. In International Journal of Psychophysiology. https://doi.org/10.1016/S0167-8760(01)00178-7

Neva, J. L., Legon, W., \& Staines, W. R. (2012). Primary motor cortex excitability is modulated with bimanual training. Neuroscience Letters, 514(2), 147-151. https://doi.org/10.1016/j.neulet.2012.02.075

Neva, J. L., Singh, A. M., Vesia, M., \& Staines, W. R. (2014). Selective modulation of left primary motor cortex excitability after continuous theta burst stimulation to right primary motor cortex and bimanual training. Behavioural Brain Research, 269, 138-146. https://doi.org/10.1016/j.bbr.2014.04.041

Neva, J. L., Vesia, M., Singh, A. M., \& Staines, W. R. (2014). Modulation of left primary motor cortex excitability after bimanual training and intermittent theta burst stimulation to left dorsal premotor cortex. Behavioural Brain Research, 261, 289-296. https://doi.org/10.1016/j.bbr.2013.12.029

Newell, K. M. (1991). Motor Skill Acquisition. Annual Review of Psychology. https://doi.org/10.1146/annurev.ps.42.020191.001241

Nudo, R J, Milliken, G. W., Jenkins, W. M., \& Merzenich, M. M. (1996). Use-dependent alterations of movement representations in primary motor cortex of adult squirrel monkeys. The Journal of Neuroscience: The Official Journal of the Society for Neuroscience. https://doi.org/8551360

Nudo, Randolph J. (2003). Adaptive plasticity in motor cortex: Implications for rehabilitation after brain injury. Journal of Rehabilitation Medicine, 35(SUPPL. 41), 7-10. 
https://doi.org/10.1080/16501960310010070

Ostadan, F., Centeno, C., Daloze, J. F., Frenn, M., Lundbye-Jensen, J., \& Roig, M. (2016). Changes in corticospinal excitability during consolidation predict acute exercise-induced off-line gains in procedural memory. Neurobiology of Learning and Memory, 136, 196203. https://doi.org/10.1016/j.nlm.2016.10.009

Panja, D., \& Bramham, C. R. (2014). BDNF mechanisms in late LTP formation: A synthesis and breakdown. Neuropharmacology. https://doi.org/10.1016/j.neuropharm.2013.06.024

Park, H., Kim, J. S., \& Chung, C. K. (2013). Differential Beta-Band Event-Related Desynchronization during Categorical Action Sequence Planning. PLoS ONE. https://doi.org/10.1371/journal.pone.0059544

Pascual-Leone, A., Wassermann, E. M., Grafman, J., \& Hallett, M. (1996). The role of the dorsolateral prefrontal cortex in implicit procedural learning. Experimental Brain Research. https://doi.org/10.1007/BF00230427

Penhune, V. B., \& Steele, C. J. (2012). Parallel contributions of cerebellar, striatal and M1 mechanisms to motor sequence learning. Behavioural Brain Research. https://doi.org/10.1016/j.bbr.2011.09.044

Percio, C. Del, Infarinato, F., Iacoboni, M., Marzano, N., Soricelli, A., Aschieri, P., ... Babiloni, C. (2010). Movement-related desynchronization of alpha rhythms is lower in athletes than non-athletes: A high-resolution EEG study. Clinical Neurophysiology. https://doi.org/10.1016/j.clinph.2009.12.004

Perez, M. A., Lundbye-Jensen, J., \& Nielsen, J. B. (2006). Changes in corticospinal drive to spinal motoneurones following visuo-motor skill learning in humans. Journal of Physiology, 573(3), 843-855. https://doi.org/10.1113/jphysiol.2006.105361 
Pollok, B., Latz, D., Krause, V., Butz, M., \& Schnitzler, A. (2014). Changes of motor-cortical oscillations associated with motor learning. Neuroscience, 275, 47-53. https://doi.org/10.1016/j.neuroscience.2014.06.008

Pozzo-Miller, L. D., Gottschalk, W., Zhang, L., McDermott, K., Du, J., Gopalakrishnan, R., ... Lu, B. (1999). Impairments in high-frequency transmission, synaptic vesicle docking, and synaptic protein distribution in the hippocampus of BDNF knockout mice. The Journal of Neuroscience: The Official Journal of the Society for Neuroscience.

https://doi.org/10.1523/JNEUROSCI.19-12-04972.1999

Quaney, B. M., Boyd, L. A., McDowd, J. M., Zahner, L. H., Jianghua He, Mayo, M. S., \& MacKo, R. F. (2009). Aerobic exercise improves cognition and motor function poststroke. Neurorehabilitation and Neural Repair. https://doi.org/10.1177/1545968309338193

Rajab, A. S., Crane, D. E., Middleton, L. E., Robertson, A. D., Hampson, M., \& MacIntosh, B. J. (2014). A single session of exercise increases connectivity in sensorimotor-related brain networks: a resting-state fMRI study in young healthy adults. Frontiers in Human Neuroscience, 8. https://doi.org/10.3389/fnhum.2014.00625

Rasmussen, P., Brassard, P., Adser, H., Pedersen, M. V., Leick, L., Hart, E., ... Pilegaard, H. (2009). Evidence for a release of brain-derived neurotrophic factor from the brain during exercise. Experimental Physiology. https://doi.org/10.1113/expphysiol.2009.048512

Redondo, R. L., \& Morris, R. G. M. (2011). Making memories last: The synaptic tagging and capture hypothesis. Nature Reviews Neuroscience. https://doi.org/10.1038/nrn2963

Robertson, E. M., Tormos, J. M., Maeda, F., \& Pascual-Leone, A. (2001). The role of the dorsolateral prefrontal cortex during sequence learning is specific for spatial information. Cerebral Cortex. https://doi.org/10.1093/cercor/11.7.628 
Roig, M., Nordbrandt, S., Geertsen, S. S., \& Nielsen, J. B. (2013). The effects of cardiovascular exercise on human memory: A review with meta-analysis. Neuroscience and Biobehavioral Reviews. https://doi.org/10.1016/j.neubiorev.2013.06.012

Roig, M., Skriver, K., Lundbye-Jensen, J., Kiens, B., \& Nielsen, J. B. (2012). A Single Bout of Exercise Improves Motor Memory. PLoS ONE, 7(9). https://doi.org/10.1371/journal.pone.0044594

Roig, M., Thomas, R., Mang, C. S., Snow, N. J., Ostadan, F., Boyd, L. A., \& Lundbye-Jensen, J. (2016). Time-Dependent Effects of Cardiovascular Exercise on Memory. Exercise and Sport Sciences Reviews. https://doi.org/10.1249/JES.0000000000000078

Sanger, T. D., Garg, R. R., \& Chen, R. (2001). Interactions between two different inhibitory systems in the human motor cortex. The Journal of Physiology. https://doi.org/PHY_11174 [pii]

Schiffer, T., Schulte, S., Sperlich, B., Achtzehn, S., Fricke, H., \& Strüder, H. K. (2011). Lactate infusion at rest increases BDNF blood concentration in humans. Neuroscience Letters. https://doi.org/10.1016/j.neulet.2010.11.035

Schmidt, R. (1975). A Schema Theory of Discrete Motor Skill Learning. Psychological Review. https://doi.org/10.1037/h0076770

Schmidt, R. A., \& Bjork, R. A. (1992). New Conceptualizations of Practice: Common Principles in Three Paradigms Suggest New Concepts for Training. Psychological Science. https://doi.org/10.1111/j.1467-9280.1992.tb00029.x

Schmidt, R., \& Lee, T. (2011). Motor Control and Learning: A Behavioral Emphasis. Human Kinetics. https://doi.org/10.1016/0021-9290(88)90286-2

Shadmehr, R., \& Holcomb, H. H. (1997). Neural correlates of motor memory consolidation. 
Science (New York, N.Y.), 277(5327), 821-825.

https://doi.org/10.1126/science.277.5327.821

Sherwood, D. E. (1996). The benefits of random variable practice for spatial accuracy and error detection in a rapid aiming task. Research Quarterly for Exercise and Sport. https://doi.org/10.1080/02701367.1996.10607923

Shibasaki, H., \& Hallett, M. (2006). What is the Bereitschaftspotential? Clinical Neurophysiology. https://doi.org/10.1016/j.clinph.2006.04.025

Singh, A. M., Duncan, R. E., Neva, J. L., \& Staines, W. R. (2014). Aerobic exercise modulates intracortical inhibition and facilitation in a nonexercised upper limb muscle. BMC Sports Science, Medicine and Rehabilitation, 6(1). https://doi.org/10.1186/2052-1847-6-23

Singh, A. M., Duncan, R. E., \& Staines, W. R. (2016). Aerobic exercise abolishes cTBS-induced suppression of motor cortical excitability. Neuroscience Letters, 633, 215-219. https://doi.org/10.1016/j.neulet.2016.09.027

Singh, A. M., Neva, J. L., \& Staines, W. R. (2014). Acute exercise enhances the response to paired associative stimulation-induced plasticity in the primary motor cortex. Experimental Brain Research, 232(11), 3675-3685. https://doi.org/10.1007/s00221-014-4049-z

Singh, A. M., Neva, J. L., \& Staines, W. R. (2016). Aerobic exercise enhances neural correlates of motor skill learning. Behavioural Brain Research, 301, 19-26. https://doi.org/10.1016/j.bbr.2015.12.020

Singh, A. M., \& Staines, W. R. (2015). The effects of acute aerobic exercise on the primary motor cortex. Journal of Motor Behavior. https://doi.org/10.1080/00222895.2014.983450

Skriver, K., Roig, M., Lundbye-Jensen, J., Pingel, J., Helge, J. W., Kiens, B., \& Nielsen, J. B. (2014). Acute exercise improves motor memory: Exploring potential biomarkers. 
Neurobiology of Learning and Memory, 116, 46-58.

https://doi.org/10.1016/j.nlm.2014.08.004

Smith, A. E., Goldsworthy, M. R., Garside, T., Wood, F. M., \& Ridding, M. C. (2014). The influence of a single bout of aerobic exercise on short-interval intracortical excitability. Experimental Brain Research, 232(6), 1875-1882. https://doi.org/10.1007/s00221-014$3879-\mathrm{z}$

Smith, A. L., \& Staines, W. R. (2010). Cortical and behavioral adaptations in response to shortterm inphase versus antiphase bimanual movement training. Experimental Brain Research, 205(4), 465-477. https://doi.org/10.1007/s00221-010-2381-5

Smith, A. L., \& Staines, W. R. (2006). Cortical adaptations and motor performance improvements associated with short-term bimanual training. Brain Research, 1071(1), 165174. https://doi.org/10.1016/j.brainres.2005.11.084

Smith, A. L., \& Staines, W. R. (2012). Externally cued inphase bimanual training enhances preparatory premotor activity. Clinical Neurophysiology, 123(9), 1846-1857. https://doi.org/10.1016/j.clinph.2012.02.060

Snow, N. J., Mang, C. S., Roig, M., McDonnell, M. N., Campbell, K. L., \& Boyd, L. A. (2016). The effect of an acute bout of moderate-intensity aerobic exercise on motor learning of a continuous tracking task. PLoS ONE, 11(2). https://doi.org/10.1371/journal.pone.0150039

Stackman, R. W., Hammond, R. S., Linardatos, E., Gerlach, A., Maylie, J., Adelman, J. P., \& Tzounopoulos, T. (2002). Small conductance Ca2+-activated K+ channels modulate synaptic plasticity and memory encoding. Journal of Neuroscience. https://doi.org/22/23/10163 [pii]\

Staines, W. R., McIlroy, W. E., Graham, S. J., \& Black, S. E. (2001). Bilateral movement 
enhances ipsilesional cortical activity in acute stroke: A pilot functional MRI study. Neurology. https://doi.org/10.1212/WNL.56.3.401

Statton, M. A., Encarnacion, M., Celnik, P., \& Bastian, A. J. (2015). A single bout of moderate aerobic exercise improves motor skill acquisition. PLOS ONE, 10(10). https://doi.org/10.1371/journal.pone.0141393

Stavrinos, E. L., \& Coxon, J. P. (2017). High-intensity interval exercise promotes motor cortex disinhibition and early motor skill consolidation. Journal of Cognitive Neuroscience. https://doi.org/10.1162/jocn_a_01078

Stinear, J. W., \& Byblow, W. D. (2002). Disinhibition in the human motor cortex is enhanced by synchronous upper limb movements. Journal of Physiology. https://doi.org/10.1113/jphysiol.2002.023986

Sun, F. T., Miller, L. M., Rao, A. A., \& D’Esposito, M. (2007). Functional connectivity of cortical networks involved in bimanual motor sequence learning. Cerebral Cortex. https://doi.org/10.1093/cercor/bhl033

Tarkka, I. M., \& Hallett, M. (1990). Cortical topography of premotor and motor potentials preceding self-paced, voluntary movement of dominant and non-dominant hands. Electroencephalography and Clinical Neurophysiology, 75(2), 36-43. https://doi.org/10.1016/0013-4694(90)90150-I

Taylor, M. J. (1978). Bereitschaftspotential during the acquisition of a skilled motor task. Electroencephalography and Clinical Neurophysiology. https://doi.org/10.1016/00134694(78)90157-8

Thacker, J. S., Middleton, L. E., McIlroy, W. E., \& Staines, W. R. (2014). The influence of an acute bout of aerobic exercise on cortical contributions to motor preparation and execution. 
Physiological Reports, 2(10). https://doi.org/10.14814/phy2.12178

Thirugnanasambandam, N., Grundey, J., Paulus, W., \& Nitsche, M. A. (2011). Dose-Dependent Nonlinear Effect of L-DOPA on Paired Associative Stimulation-Induced Neuroplasticity in Humans. Journal of Neuroscience, 31(14), 5294-5299. https://doi.org/10.1523/JNEUROSCI.6258-10.2011

Thomas, R., Flindtgaard, M., Skriver, K., Geertsen, S. S., Christiansen, L., Korsgaard Johnsen, L., ... Lundbye-Jensen, J. (2016). Acute exercise and motor memory consolidation: Does exercise type play a role? Scandinavian Journal of Medicine \& Science in Sports. https://doi.org/10.1111/sms.12791

Thomas, R., Beck, M. M., Lind, R. R., Korsgaard Johnsen, L., Geertsen, S. S., Christiansen, L., ... Lundbye-Jensen, J. (2016). Acute Exercise and Motor Memory Consolidation: The Role of Exercise Timing. Neural Plasticity, 2016. https://doi.org/10.1155/2016/6205452

Thomas, R., Johnsen, L. K., Geertsen, S. S., Christiansen, L., Ritz, C., Roig, M., \& LundbyeJensen, J. (2016). Acute exercise and motor memory consolidation: The role of exercise intensity. PLoS ONE, 11(7). https://doi.org/10.1371/journal.pone.0159589

Tomporowski, P. D. (2003). Effects of acute bouts of exercise on cognition. Acta Psychologica. https://doi.org/10.1016/S0001-6918(02)00134-8

Tunovic, S., Press, D. Z., \& Robertson, E. M. (2014). A Physiological Signal That Prevents Motor Skill Improvements during Consolidation. Journal of Neuroscience. https://doi.org/10.1523/JNEUROSCI.3497-13.2014

Tzagarakis, C., Ince, N. F., Leuthold, A. C., \& Pellizzer, G. (2010). Beta-Band Activity during Motor Planning Reflects Response Uncertainty. Journal of Neuroscience. https://doi.org/10.1523/jneurosci.6026-09.2010 
Walter, W. G., Cooper, R., Aldridge, V. J., McCallum, W. C., \& Winter, W. G. (1964). Contingent negative variation: An electrical sign of sensorimotor association and expectancy in the human brain. Nature, 203, 380-384. https://doi.org/10.1038/203380a0

Winter, B., Breitenstein, C., Mooren, F. C., Voelker, K., Fobker, M., Lechtermann, A., ... Knecht, S. (2007). High impact running improves learning. Neurobiology of Learning and Memory, 87(4), 597-609. https://doi.org/10.1016/j.nlm.2006.11.003

Wright, D. J., Holmes, P. S., \& Smith, D. (2011). Using the movement-related cortical potential to study motor skill learning. Journal of Motor Behavior. https://doi.org/10.1080/00222895.2011.557751

Wulf, G. (1991). The Effect of Type of Practice on Motor Learning in Children. Applied Cognitive Psychology, 5, 123-134.

Xu, T., Yu, X., Perlik, A. J., Tobin, W. F., Zweig, J. A., Tennant, K., ... Zuo, Y. (2009). Rapid formation and selective stabilization of synapses for enduring motor memories. Nature. https://doi.org/10.1038/nature08389

Ying, S., Futter, M., Rosenblum, K., Webber, M. J., Hunt, S. P., Bliss, T. V. P., \& Bramham, C. R. (2002). Brain-Derived Neurotrophic Factor Induces Long-Term Potentiation in Intact Adult Hippocampus : Requirement for ERK Activation Coupled to CREB and Upregulation of Arc Synthesis. The Journal of Neuroscience. https://doi.org/22/5/1532 [pii]

Ziemann, U., Iliac', T., Pauli, C., Meintzschel, F., \& Ruge, D. (2004). Learning Modifies Subsequent Induction of Long-Term Potentiation-Like and Long-Term Depression-Like Plasticity in Human Motor Cortex. Journal of Neuroscience. https://doi.org/10.1523/JNEUROSCI.5016-03.2004 